\title{
Preparation of 1-Thio Uronic Acid Lactones and Their Use in Oligosaccharide Synthesis
}

Leendert J. van den Bos, Remy E.J.N. Litjens, Richard, J.B.H.N. van den Berg, Herman S. Overkleeft, Gijsbert A. van der Marel*

Leiden Institute of Chemistry, Leiden University, P.O. Box 9502, 2300 RA Leiden,The Netherlands, g.marel@chem.leidenuniv.nl

General Procedures: ${ }^{1} \mathrm{H}$ and ${ }^{13} \mathrm{C}$ NMR spectra were recorded on a Brüker AV-400 (400/100 MHz) and a Brüker DMX-600 (600/150 MHz) spectrometer. Chemical shifts ( $\delta$ ) are given in ppm relative to tetramethylsilane as internal standard. Coupling constants are given in Hz. All given ${ }^{13} \mathrm{C}$ spectra are proton decoupled. Mass spectra were recorded with PE/SCIEX API 165 with electronspray interface and Q-Star Applied Biosystems Q-TOF (TOF-section). Optical rotations were measured on a Propol automatic polarimeter. IR-spectra were recorded on a Shimadzu FTIR-8300. Traces of water in the donor and acceptor glycosides, diphenylsulfoxide and TTBP were removed by co-evaporation with toluene. TTBP was synthesized as described by Crich et al. ${ }^{\mathrm{i}}$ Dichloromethane (DCM, Baker p.a.) was boiled under reflux over $\mathrm{P}_{2} \mathrm{O}_{5}$ for $2 \mathrm{~h}$ and distilled immediately prior to use. Trifluoromethanesulfonic anhydride was distilled from $\mathrm{P}_{2} \mathrm{O}_{5}$. Molecular sieves $3 \AA$ were flame dried before use. Solvents used for flash chromatography and TLC were of technical grade and distilled before use. Flash chromatography was performed on Fluka silica gel 60 (0.04-0.063 mm). TLC-analysis was conducted on DC-alufolien (Merck, Kieselgel60, $\mathrm{F}_{254}$ ) with detection by UV-absorption (254 nm) were applicable and by spraying with $20 \%$ sulfuric acid in ethanol followed by charring at $\sim 150^{\circ} \mathrm{C}$ or by spraying with a solution of $\left(\mathrm{NH}_{4}\right)_{6} \mathrm{Mo}_{7} \mathrm{O}_{24} \cdot \mathrm{H}_{2} \mathrm{O}(25 \mathrm{~g} / \mathrm{l})$ and $\left(\mathrm{NH}_{4}\right)_{4} \mathrm{Ce}\left(\mathrm{SO}_{4}\right)_{4} \cdot 2 \mathrm{H}_{2} \mathrm{O}(10 \mathrm{~g} / \mathrm{l})$ in $10 \%$ sulfuric acid in ethanol followed by charring at $\sim 150^{\circ} \mathrm{C}$. 


\section{General Procedure for the TEMPO/BAIB Mediated Tandem Oxidation/Lactonization:}

To a vigorously stirred solution of $0.3 \mathrm{mmol}$ thioglycoside in $1 \mathrm{ml} \mathrm{DCM}$ and $0.5 \mathrm{ml} \mathrm{H}_{2} \mathrm{O}$ was added 0.06 mmol TEMPO (0.2 equiv.) and 0.75 mmol BAIB (2.5 equiv.). Stirring was allowed until TLC indicated complete conversion of the starting material to a higher running spot ( $15 \mathrm{~min})$. The reaction mixture was quenched by the addition of $10 \mathrm{ml} \mathrm{Na}_{2} \mathrm{~S}_{2} \mathrm{O}_{3}$ solution $\left(10 \%\right.$ in $\left.\mathrm{H}_{2} \mathrm{O}\right)$. The mixture was then extracted twice with EtOAc $(10 \mathrm{ml})$ and the combined organic layers were dried $\left(\mathrm{MgSO}_{4}\right)$, filtered and concentrated. Flash column chromatography using EtOAc/petroleum ether) afforded the pure lactones.

\section{General Procedure for Glycosidations using $\mathrm{Ph}_{2} \mathrm{SO} / \mathrm{Tf}_{2} \mathrm{O}$ :}

A solution of lactone (1 equiv), diphenyl sulfoxide (1.3 equiv.) and tri-tert-butylpyrimidine (2.5 equiv.) in DCM $(0.05 \mathrm{M})$ was stirred over activated MS3 $\AA$ for $30 \mathrm{~min}$. The mixture was cooled to $-60^{\circ} \mathrm{C}$ before triflic acid anhydride (1.3 equiv.) was added. The mixture was allowed to warm to $-50^{\circ} \mathrm{C}$ in $15 \mathrm{~min}$ followed by addition of acceptor (1.5 equiv.) in DCM (0.15M). Stirring was continued and the reaction mixture was allowed to warm to $0^{\circ} \mathrm{C}$. The reaction mixture was diluted with EtOAc and washed with water. The water/layer wash extracted twice with EtOAc. The collected organic layers were dried $\left(\mathrm{MgSO}_{4}\right)$, filtered and concentrated under reduced pressure. Flash chromatography (EtOAc/PE) and removal of the eluent afforded the corresponding title compound.

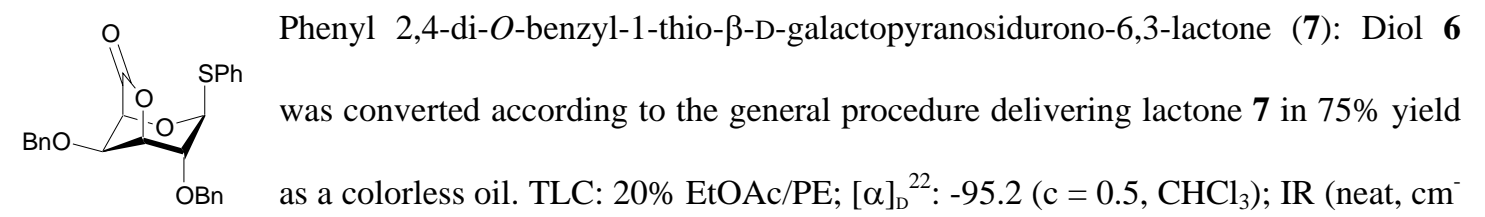
$\left.{ }^{1}\right)$ : 979, 1026, 1060, 1097, 1151, 1365, 1454, 1799, 2869, 3030; ${ }^{1} \mathrm{H}$ NMR $\left(400 \mathrm{MHz}, \mathrm{CDCl}_{3}\right) \delta=4.02$ (s, 1H, H-4), 4.23 (d, 1H, J = 4.8 Hz, H-2), 4.36 (s, 1H, H-5), 4.48 (d, 1H, $J=11.8 \mathrm{~Hz}, \mathrm{CHHBn}$ ), 4.51 (s, 2H, $\mathrm{CH}_{2} \mathrm{Bn}$ ), 4.56 (d, 1H, $J=11.8 \mathrm{~Hz}, \mathrm{CHHBn}$ ), 4.79 (d, 1H, $J=4.4 \mathrm{~Hz}, \mathrm{H}-3$ ), 5.40 (s, 1H, H-1), $7.14-7.43$ (m, 15H, H Arom); ${ }^{13} \mathrm{C}$ NMR (100 MHz, $\left.\mathrm{CDCl}_{3}\right) \delta=70.6$ (C-4), $71.1\left(\mathrm{CH}_{2} \mathrm{Bn}\right), 72.7\left(\mathrm{CH}_{2}\right.$ Bn), 75.7 (C-5), 78.4 (C-2), 78.6 (C-3), 85.6 (C-1), 127.6 - 132.4 (CH Arom), 133.5 (C $\left.\mathrm{C}_{\mathrm{q}} \mathrm{SPh}\right), 136.4$ $\left(\mathrm{C}_{\mathrm{q}} \mathrm{Bn}\right), 136.5\left(\mathrm{C}_{\mathrm{q}} \mathrm{Bn}\right), 172.5(\mathrm{C}=\mathrm{O})$; HRMS: $\left(\mathrm{M}+\mathrm{NH}_{4}\right)$ calcd for $\mathrm{C}_{26} \mathrm{H}_{24} \mathrm{O}_{5} \mathrm{~S}$ 466.16882, found 466.16946. 
HO $\underbrace{\underbrace{\mathrm{BnO}}_{\text {COOMe }}}_{\mathrm{OBn}}$ Methyl (phenyl 2,4-di-O-benzyl-1-thio- $\beta$-D-galactopyranoside) uronate (8): The mixture was stirred for 3h after TLC-analysis indicated complete conversion. The reaction mixture was neutralized upon addition of $\mathrm{Et}_{3} \mathrm{~N}$ followed by concentration under reduced pressure. Flash chromatography and removal of the eluent afforded the title compound $\mathbf{8}$ as a colorless oil in quant. yield. TLC: 20\% EtOAc/PE; IR (neat, $\mathrm{cm}^{-1}$ ): 1026, 1103, 1348, 1438, 1735, 2858; ${ }^{1} \mathrm{H}$ NMR (400 MHz, $\left.\mathrm{CDCl}_{3}\right) \delta=2.26(\mathrm{~d}, 1 \mathrm{H}, J=4.8 \mathrm{~Hz}, \quad \mathrm{OH}-3), 3.70(\mathrm{t}, 1 \mathrm{H}, J=8.8 \mathrm{~Hz}, \mathrm{H}-2), 3.73\left(\mathrm{~m}, 4 \mathrm{H}, \mathrm{H}-3\right.$ and $\mathrm{CH}_{3}$ COOMe), 4.10 (s, 1H, H-5), 4.23 (d, 1H, J = 1.2 Hz, H-4), 4.57 (d, 1H, J = 8.8 Hz, H-1), 4.63 (d, 2H, $J=11.2 \mathrm{~Hz}, 2 x C H H B n), 4.70$ (d, 1H, $J=11.2 \mathrm{~Hz}, \mathrm{CHHBn}), 4.91$ (d, 1H, $J=11.2 \mathrm{~Hz}, \mathrm{CHHBn}), 7.24-$ 7.66 (m, 15H, CH Arom); ${ }^{13} \mathrm{C}$ NMR (100 MHz, $\left.\mathrm{CDCl}_{3}\right) \delta=52.4\left(\mathrm{CH}_{3} \mathrm{COOMe}\right), 74.8\left(\mathrm{CH}_{2} \mathrm{Bn}\right), 75.3$ ( $\left.\mathrm{CH}_{2} \mathrm{Bn}\right), 75.3$ (C-3), 77.0 (C-5), 77.3 (C-4), 77.5 (C-2), 87.5 (C-1), 127.6132 .3 (CH Arom), 133.5 $\left(\mathrm{C}_{\mathrm{q}} \mathrm{SPh}\right), 137.9\left(\mathrm{C}_{\mathrm{q}} \mathrm{Bn}\right), 138.0\left(\mathrm{C}_{\mathrm{q}} \mathrm{Bn}\right), 168.4(\mathrm{C}=\mathrm{O})$; HRMS: $(\mathrm{M}+\mathrm{Na})$ calcd for $\mathrm{C}_{27} \mathrm{H}_{28} \mathrm{O}_{6} \mathrm{~S}$ 503.14988, found 503.15082.

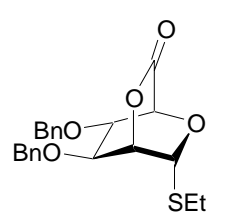

Ethyl 3,4-di-O-benzyl-1-thio- $\alpha$-D-mannopyranosidurono-6,2-lactone (10): Diol 9 was converted according to the general procedure delivering lactone $\mathbf{1 0}$ in $77 \%$ yield as a colorless oil. TLC: $20 \% \mathrm{EtOAc/PE} ;[\alpha]_{\mathrm{D}}^{22}:+151.6\left(\mathrm{c}=1.7, \mathrm{CHCl}_{3}\right)$; IR (neat, $\mathrm{cm}^{-1}$ ):

981, 1064, 1188, 1352, 1454, 1672, 1778, 2871, 3031, 3192; ${ }^{1} \mathrm{H}$ NMR $\left(300 \mathrm{MHz}, \mathrm{CDCl}_{3}\right) \delta=1.34(\mathrm{t}$, 3H, $J=7.4 \mathrm{~Hz}, \mathrm{CH}_{3} \mathrm{SEt}$ ), 2.77 (m, 2H, $\mathrm{CH}_{2} \mathrm{SEt}$ ), 3.80 (s, 1H, H-4), 4.07 (s, 1H, H-3), 4.47 (d, 1H, $J=$ $11.2 \mathrm{~Hz}, \mathrm{CH} H \mathrm{Bn}), 4.49$ (s, 1H, H-5), 4.50 (d, 1H, $J=12.4 \mathrm{~Hz}, \mathrm{CH} H \mathrm{Bn}), 4.61$ (d, 1H, $J=11.2 \mathrm{~Hz}$, CHHBn), 4.65 (d, 1H, $J=12.4$ Hz, CHHBn), 4.85 (s, 1H, H-2), 5.27 (d, 1H, J = 2.6 Hz, H-1), $7.30-$ 7.36 (m, 10H, H Arom); ${ }^{13} \mathrm{C}$ NMR $\left(75 \mathrm{MHz}, \mathrm{CDCl}_{3}\right) \delta=16.1\left(\mathrm{CH}_{3} \mathrm{SEt}\right), 25.8\left(\mathrm{CH}_{2} \mathrm{SEt}\right), 71.8\left(\mathrm{CH}_{2}\right.$ Bn), 71.9 ( $\left.\mathrm{CH}_{2} \mathrm{Bn}\right)$, 72.9, 76.3, 77.9, 80.6 (C-2, C-3, C-4, C-5), 81.6 (C-1), 128.7 - 129.4 (CH Arom), 137.6 $\left(\mathrm{C}_{\mathrm{q}} \mathrm{Bn}\right), 137.7\left(\mathrm{C}_{\mathrm{q}} \mathrm{Bn}\right), 168.8(\mathrm{C}=\mathrm{O})$; HRMS: $\left(\mathrm{M}+\mathrm{NH}_{4}\right)$ calcd for $\mathrm{C}_{22} \mathrm{H}_{24} \mathrm{O}_{5} \mathrm{~S}: 418.16827$, found 418.16864.

SEt Compound 10 was suspended in $\mathrm{MeOH}$ and stirred at reflux temperature for $5 \mathrm{~h}$ after which TLC-analysis indicated complete conversion. Removal of the methanol under reduced pressure 
and co-evaporation of the residual oil afforded the title compound $\mathbf{1 1}$ (quant.) as a light yellow oil. TLC: $20 \%$ EtOAc/PE; $[\alpha]_{\mathrm{D}}^{22}:+72.0\left(\mathrm{c}=1, \mathrm{CHCl}_{3}\right.$ ); IR (neat, $\left.\mathrm{cm}^{-1}\right)$ : 702, 732, 1033, 1087, 1234, 1373, 1735; ${ }^{1} \mathrm{H}$ NMR (400 MHz, $\left.\mathrm{CDCl}_{3}\right) \delta=1.31$ (t, 3H, $\left.J=7.4 \mathrm{~Hz}, \mathrm{CH}_{3} \mathrm{SEt}\right), 2.71$ (m, 2H, $\mathrm{CH}_{2} \mathrm{SEt}$ ), 3.64 (s, 3H, $\mathrm{CH}_{3} \mathrm{COOMe}$ ), 3.84 (dd, $1 \mathrm{H}, J=7.0 \mathrm{~Hz}, J=3.0 \mathrm{~Hz}, \mathrm{H}-3$ ), 4.00 (dd, $1 \mathrm{H}, J=5.2, J=3.0 \mathrm{~Hz}, \mathrm{H}-$ 2), 4.16 (t, $1 \mathrm{H}, J=7.0 \mathrm{~Hz}, J=6.2 \mathrm{~Hz}, \mathrm{H}-4$ ), 4.56 (d, $1 \mathrm{H}, J=6.2 \mathrm{~Hz}, \mathrm{H}-5), 4.61$ (s, 2H, $\mathrm{CH}_{2} \mathrm{Bn}$ ), 4.64 (d, $1 \mathrm{H}, J=11.6 \mathrm{~Hz}, \mathrm{CHHBn}), 4.68$ (d, 1H, $J=11.6 \mathrm{~Hz}, \mathrm{CHHBn}), 5.35$ (d, 1H, $J=5.2 \mathrm{~Hz}, \mathrm{H}-1$ ); 7.24 7.35 (m, 10H, CH Arom); ${ }^{13} \mathrm{C}$ NMR (100 MHz, $\left.\mathrm{CDCl}_{3}\right) \delta=14.9\left(\mathrm{CH}_{3} \mathrm{SEt}\right), 25.5\left(\mathrm{CH}_{2} \mathrm{SEt}\right), 52.2\left(\mathrm{CH}_{3}\right.$ COOMe), 68.3 (C-2), 71.8 (C-5), 72.5 ( $\left.\mathrm{CH}_{2} \mathrm{Bn}\right), 73.7\left(\mathrm{CH}_{2} \mathrm{Bn}\right), 75.4$ (C-4), 78.6 (C-3), 84.5 (C-1), 127.7 - 128.5 (CH Arom), $137.3\left(\mathrm{C}_{\mathrm{q}} \mathrm{Bn}\right), 137.7\left(\mathrm{C}_{\mathrm{q}} \mathrm{Bn}\right), 169.7(\mathrm{C}=\mathrm{O})$; HRMS: $\left(\mathrm{M}+\mathrm{NH}_{4}\right)$ calcd for $\mathrm{C}_{23} \mathrm{H}_{28} \mathrm{O}_{6} \mathrm{~S}: 450.19503$, found 450.19748 .

$\mathrm{O}$ Ethyl 2-O-acetyl-4-O-benzyl-1-thio- $\alpha$-D-mannopyranosidurono-6,3-lactone (13): Diol

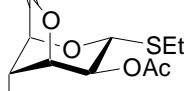
12 was converted according to the general procedure delivering lactone $\mathbf{1 3}$ in $72 \%$ $\mathrm{OBn}$ yield as a colorless oil. TLC: $20 \% \mathrm{EtOAc} / \mathrm{PE} ;[\alpha]_{\mathrm{D}}{ }^{22}:+40.0\left(\mathrm{c}=0.5, \mathrm{CHCl}_{3}\right)$; IR (neat, $\left.\mathrm{cm}^{-1}\right): 948,1012,1058,1161,1224,1373,1456,1741,1799,2873 ;{ }^{1} \mathrm{H}$ NMR $\left(400 \mathrm{MHz}, \mathrm{CDCl}_{3}\right) \delta=$ 1.25 (t, 3H, $J=7.2 \mathrm{~Hz}, \mathrm{CH}_{3} \mathrm{SEt}$ ), 2.13 (s, 3H, $\mathrm{CH}_{3} \mathrm{Ac}$ ), 2.71 (m, 2H, $\mathrm{CH}_{2} \mathrm{SEt}$ ), 4.07 (dd, 1H, $J=5.9$ $\mathrm{Hz}, J=2.8 \mathrm{~Hz}, \mathrm{H}-4), 4.28$ (dd, 1H, $J=2.8 \mathrm{~Hz}, J=1.0 \mathrm{~Hz}, \mathrm{H}-5$ ), 4.63 (d, $1 \mathrm{H}, J=11.8 \mathrm{~Hz}, \mathrm{CH} H \mathrm{Bn}$ ), 4.69 (d, 1H, $J=9.2 \mathrm{~Hz}, \mathrm{H}-1), 4.75$ (d, 1H, $J=11.8 \mathrm{~Hz}, \mathrm{CHHBn}$ ), 4.79 (dt, 1H, $J=5.9 \mathrm{~Hz}, J=1.2 \mathrm{~Hz}$, $J=1.0 \mathrm{~Hz}, \mathrm{H}-3$ ), 5.40 (dd, $1 \mathrm{H}, J=1.2 \mathrm{~Hz}, \mathrm{H}-2), 7.26-7.40$ (m, 5H, CH Arom); ${ }^{13} \mathrm{C}$ NMR (100 MHz, $\left.\mathrm{CDCl}_{3}\right) \delta=14.8\left(\mathrm{CH}_{3} \mathrm{SEt}\right), 20.8\left(\mathrm{CH}_{3} \mathrm{Ac}\right), 23.9\left(\mathrm{CH}_{2} \mathrm{SEt}\right), 67.3(\mathrm{C}-2), 72.0\left(\mathrm{CH}_{2} \mathrm{Bn}\right), 72.1(\mathrm{C}-5)$, 74.8 (C-4), 77.5 (C-3), 80.3 (C-1), 128.1 - 128.7 (CH Arom), 136.0 ( $\left.\mathrm{C}_{\mathrm{q}} \mathrm{Bn}\right), 169.5$ (C=O Ac or lactone), 169.6 ( $\mathrm{C}=\mathrm{O}$ lactone or Ac); HRMS: $\left(\mathrm{M}+\mathrm{NH}_{4}\right)$ calcd for $\mathrm{C}_{17} \mathrm{H}_{20} \mathrm{O}_{6} \mathrm{~S}$ : 370.13243, found 370.12914.

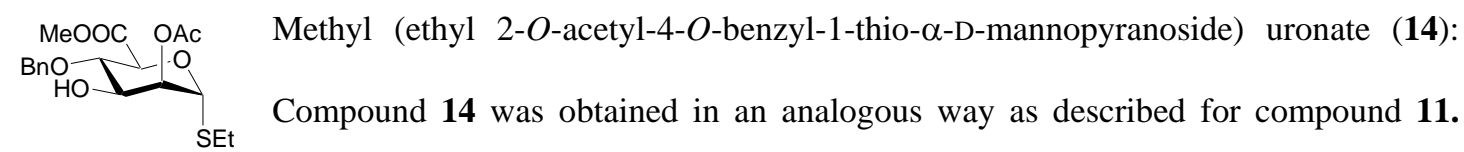
TLC: $20 \%$ EtOAc/PE; $[\alpha]_{\mathrm{D}}^{22}:+35.0\left(\mathrm{c}=0.6, \mathrm{CHCl}_{3}\right)$; IR (neat, $\left.\mathrm{cm}^{-1}\right): 1035,1091,1232,1373,1438$, 1739, 2858; ${ }^{1} \mathrm{H}$ NMR $\left(400 \mathrm{MHz}, \mathrm{CDCl}_{3}\right) \delta=1.30$ (t, $1 \mathrm{H}, J=7.6 \mathrm{~Hz}, \mathrm{CH}_{3} \mathrm{SEt}$ ), 2.16 (s, 3H, $\mathrm{CH}_{3} \mathrm{Ac}$ ), $2.65\left(\mathrm{CH}_{2} \mathrm{SEt}\right.$ ), 3.78 (s, 3H, $\mathrm{CH}_{3} \mathrm{COOMe}$ ), 3.98 (t, $\left.1 \mathrm{H}, J=8.8 \mathrm{~Hz}, \mathrm{H}-4\right), 4.08$ (dd, $1 \mathrm{H}, J=8.8 \mathrm{~Hz}, J=$ $8.4 \mathrm{~Hz}, J=3.3 \mathrm{~Hz}, \mathrm{H}-3$ ), 4.58 (d, 1H, $J=8.4 \mathrm{~Hz}, \mathrm{H}-5), 4.68$ (d, 1H, $J=11.2 \mathrm{~Hz}, \mathrm{CHHBn}$ ), 4.72 (d, 1H, 
$J=11.2 \mathrm{~Hz}, \mathrm{CHHBn}), 5.20$ (t, 1H, $J=3.3, J=2.8 \mathrm{~Hz}, \mathrm{H}-2), 3.35$ (d, 1H, $J=2.8 \mathrm{~Hz}, \mathrm{H}-1$ ), $7.26-7.37$ (m, 5H, CH Arom); ${ }^{13} \mathrm{C}$ NMR (100 MHz, $\left.\mathrm{CDCl}_{3}\right) \delta=14.8\left(\mathrm{CH}_{3} \mathrm{SEt}\right), 22.4\left(\mathrm{CH}_{3} \mathrm{Ac}\right), 25.1\left(\mathrm{CH}_{2} \mathrm{SEt}\right)$, 52.5 ( $\left.\mathrm{CH}_{3} \mathrm{COOMe}\right), 70.1$ (C-3), 71.3 (C-5), 72.8 (C-2), 74.5 ( $\left.\mathrm{CH}_{2} \mathrm{Bn}\right), 77.4$ (C-4), 81.9 (C-1), 127.8 128.5 ( $\mathrm{CH}$ Arom), 137.8 ( $\mathrm{C}_{\mathrm{q}} \mathrm{Bn}$ ), 169.8 ( $\mathrm{C}=\mathrm{O}$ Ac or lactone), 170.5 ( $\mathrm{C}=\mathrm{O}$ lactone or Ac); HRMS: (M $+\mathrm{NH}_{4}$ ) calcd for $\mathrm{C}_{18} \mathrm{H}_{24} \mathrm{O}_{7} \mathrm{~S} 402.15865$, found 402.16217 .

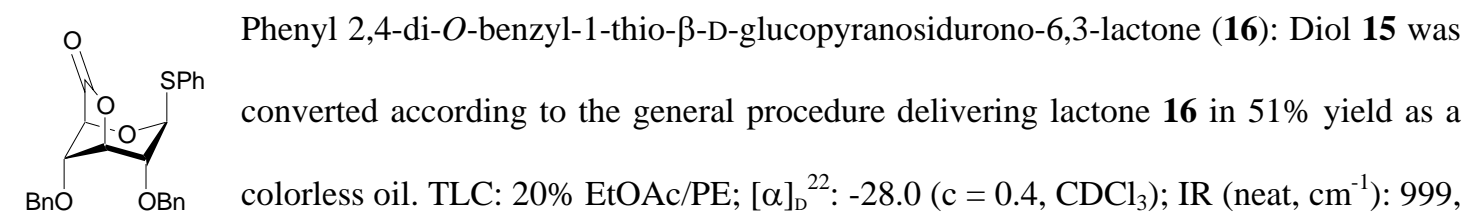
1024, 1078, 1367, 1454, 1585, 1637, 1801, 3006, 3223; ${ }^{1} \mathrm{H}$ NMR $\left(400 \mathrm{MHz}, \mathrm{CDCl}_{3}\right) \delta=3.83(\mathrm{~d}, 1 \mathrm{H}$, $J=5.4 \mathrm{~Hz}, \mathrm{H}-2), 4.06$ (t, 1H, $J=3.2 \mathrm{~Hz}, J=2.4 \mathrm{~Hz}, J=1.0 \mathrm{~Hz}, \mathrm{H}-4), 4.10(\mathrm{~d}, 1 \mathrm{H}, J=2.4 \mathrm{~Hz}, \mathrm{H}-5)$, 4.56 (d, $1 \mathrm{H}, J=11.6 \mathrm{~Hz}, \mathrm{CH} H \mathrm{Bn}), 4.60$ (d, 1H, $J=11.6 \mathrm{~Hz}, \mathrm{CHHBn}$ ), 4.64 (dd, $1 \mathrm{H}, J=3.2 \mathrm{~Hz}, J=$ $1.0 \mathrm{~Hz}, \mathrm{H}-3$ ), 4.68 (d, 1H, $J=11.6 \mathrm{~Hz}, \mathrm{CHHBn}), 4.70$ (d, 1H, $J=11.6 \mathrm{~Hz}, \mathrm{CHHBn}), 5.61$ (d, 1H, $J=$ $5.4 \mathrm{~Hz}, \mathrm{H}-1) ;{ }^{13} \mathrm{C}$ NMR (100 MHz, $\left.\mathrm{CDCl}_{3}\right) \delta=69.7$ (C-5), $72.2\left(\mathrm{CH}_{2} \mathrm{Bn}\right), 73.4\left(\mathrm{CH}_{2} \mathrm{Bn}\right), 74.5(\mathrm{C}-4)$, 74.7 (C-3), 76.9 (C-2), 84.4 (C-1), 128.0 - 133.2 (CH Arom), 132.3 ( $\left.\mathrm{C}_{\mathrm{q}} \mathrm{SPh}\right), 136.3\left(\mathrm{C}_{\mathrm{q}} \mathrm{Bn}\right), 137.0\left(\mathrm{C}_{\mathrm{q}}\right.$ $\mathrm{Bn}), 170.5(\mathrm{C}=\mathrm{O})$; HRMS: $\left(\mathrm{M}+\mathrm{NH}_{4}\right)$ calcd for $\mathrm{C}_{26} \mathrm{H}_{24} \mathrm{O}_{5} \mathrm{~S}$ 466.16882, found 466.17197.

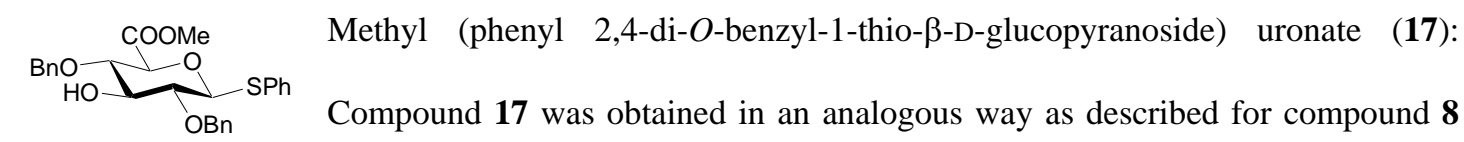
TLC: 20\% EtOAc/PE; IR (neat, $\mathrm{cm}^{-1}$ ): 1026, 1074, 1207, 1438, 1747, 2920; ${ }^{1} \mathrm{H}$ NMR (400 MHz, $\left.\mathrm{CDCl}_{3}\right) \delta=3.92(\mathrm{dd}, 1 \mathrm{H}, J=9.6 \mathrm{~Hz}, J=8.8 \mathrm{~Hz}, \mathrm{H}-2), 3.72(\mathrm{t}, 1 \mathrm{H}, J=9.2 \mathrm{~Hz}, J=8.8 \mathrm{~Hz}, \mathrm{H}-4), 3.75$ (s, 3H, $\mathrm{CH}_{3} \mathrm{COOMe}$ ), 3.78 (t, 1H, $J=9.2 \mathrm{~Hz}, J=8.8 \mathrm{~Hz}, \mathrm{H}-3$ ), 3.91 (d, 1H, $J=9.2 \mathrm{~Hz}, \mathrm{H}-5$ ), 4.63 (d, 1H, $J=11.2 \mathrm{~Hz}, \mathrm{CHHBn}$ ), 4.66 (d, 1H, $J=9.6 \mathrm{~Hz}, \mathrm{H}-1$ ), 4.68 (d, 1H, $J=10.4 \mathrm{~Hz}, \mathrm{CHHBn}$ ), 4.76 (d, $1 \mathrm{H}, J=11.2 \mathrm{~Hz}, \mathrm{CHHBn}), 4.96$ (d, 1H, $J=10.4 \mathrm{~Hz}, \mathrm{CHHBn}), 7.25-7.55$ (m, 15H. CH Arom); ${ }^{13} \mathrm{C}$ NMR (100 MHz, $\left.\mathrm{CDCl}_{3}\right) \delta=52.5\left(\mathrm{CH}_{3} \mathrm{COOMe}\right), 74.8\left(\mathrm{CH}_{2} \mathrm{Bn}\right), 75.2\left(\mathrm{CH}_{2} \mathrm{Bn}\right), 77.6(\mathrm{C}-5), 77.7(\mathrm{C}-$ 3), 78.6 (C-4), 80.0 (C-2), 88.0 (C-1), 127.8 - 132.0 (CH Arom); HRMS: (M + $\mathrm{NH}_{4}$ ) calcd for $\mathrm{C}_{27} \mathrm{H}_{28} \mathrm{O}_{6} \mathrm{~S}: 498.19503$, found 498.19424 . 
$\mathrm{O}$ OBn

OBn 2,3,4-Tri-O-benzyl- $\beta$-D-glucopyranosidurono-6,1-lactone (19): Diol 18 was converted according to the general procedure delivering lactone 19 in 27\% yield as a colorless oil. OBn TLC: $20 \%$ EtOAc/PE; $[\alpha]_{\mathrm{D}}^{22}:-4.6\left(\mathrm{c}=0.2, \mathrm{CHCl}_{3}\right)$; IR (neat, $\left.\mathrm{cm}^{-1}\right)$ : 912, 1043, 1068, 1178, 1454, 1807, 2866, 3340; ${ }^{1} \mathrm{H}$ NMR (400 MHz, $\left.\mathrm{CDCl}_{3}\right) \delta=3.50$ (s, 1H, H-2), 3.63 (s, $1 \mathrm{H}, \mathrm{H}-4$ or H-3), 3.68 (t, 1H, $J=1.6 \mathrm{~Hz}, \mathrm{H}-3$ or H-4), 4.26 (d, $1 \mathrm{H}, J=12.4 \mathrm{~Hz}, \mathrm{CHHBn}$ ), 4.40 (d, $1 \mathrm{H}, J=12.4 \mathrm{~Hz}$, CHHBn), 4.47 (d, 1H, $J=12.4$ Hz, CHHBn), 4.54 (d, 1H, $J=12.4 \mathrm{~Hz}, \mathrm{CHHBn}$ ), 4.59 (d, 1H, $J=12.4$ Hz, CHHBn), 4.64 (d, 1H, J = 12.4 Hz, CHHBn), 5.79 (s, 1H, H-1), 7.15 - 7.36 (m, 15H, CH Arom); ${ }^{13} \mathrm{C}$ NMR (100 MHz, $\left.\mathrm{CDCl}_{3}\right) \delta=71.6\left(\mathrm{CH}_{2} \mathrm{Bn}\right), 72.0\left(\mathrm{CH}_{2} \mathrm{Bn}\right), 72.1$ (C-5), 72.4 (C-4 or C-3), 72.4 ( $\mathrm{CH}_{2} \mathrm{Bn}$ ), 73.0 (C-2), 75.0 (C-3 or C-4), 102.5 (C-1), 127.7 - 128.6 (CH Arom), 136.9 (C $\mathrm{C}_{\mathrm{q}} \mathrm{Bn}$ ), 170.0 (C=O); HRMS: $\left(\mathrm{M}+\mathrm{Na}\right.$ ) calcd for $\mathrm{C}_{27} \mathrm{H}_{26} \mathrm{O}_{6}$ 469.16216, found 469.15979 .

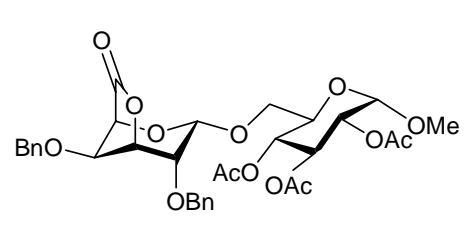

Methyl Donor $\mathbf{7}$ was glycosidated with acceptor $\mathbf{2 0}$ in the same way as described in the general procedure delivering the title compound $\mathbf{2 1}$ as a colorless oil. TLC: 30\% EtOAc/PE; $[\alpha]_{\mathrm{D}}^{22}:+82.0\left(\mathrm{c}=1, \mathrm{CHCl}_{3}\right.$ ); IR (neat, $\left.\mathrm{cm}^{-1}\right)$ : 925, 1028, 1159, 1217, 1367, 1454, 1747, 1799, 2920; ${ }^{1} \mathrm{H}$ NMR (400 MHz, $\left.\mathrm{CDCl}_{3}\right) \delta=1.94$ (s, 6H, 2xCH $\mathrm{Ac}$ ), 2.00 (s, 3H, $\mathrm{CH}_{3} \mathrm{Ac}$ ), 3.28 (s, 3H, $\mathrm{CH}_{3} \mathrm{OMe}$ ), 3.62 (d, 1H, $J=11.6 \mathrm{~Hz}, \mathrm{H}-6$ ), 3.84 (bd, 1H, $J=10.4$ Hz, H-5), 3.89 - 3.94 (m, 2H, H6 and H-2'), 4.05 (s, 1H, H-4'), 4.44 (s, 1H, H-5'), 4.51 (s, 2H, $\mathrm{CH}_{2} \mathrm{Bn}$ ), 4.53 (d, 1H, $J=12.0 \mathrm{~Hz}$, CHHBn), 4.62 (d, 1H, $J=4.0$ Hz, H-3'), 4.74 (dd, 1H, $J=10.2 \mathrm{~Hz}, J=3.6 \mathrm{~Hz}, \mathrm{H}-2$ ), 4.81 (s, 1H, H1'), 4.83 (d, 1H, $J=3.2 \mathrm{~Hz}, \mathrm{H}-1), 4.86$ (d, 1H, $J=12.0 \mathrm{~Hz}, \mathrm{CHHBn}$ ), 5.04 (t, 1H, $J=9.6 \mathrm{~Hz}, \mathrm{H}-4$ ), 5.38 (t, $1 \mathrm{H}, J=10.2 \mathrm{~Hz}, J=9.6 \mathrm{~Hz}, \mathrm{H}-3), 7.19-7.48$ (m, 10H, CH Arom); ${ }^{13} \mathrm{C}$ NMR (100 MHz, $\left.\mathrm{CDCl}_{3}\right) \delta=20.6\left(3 \mathrm{xCH}_{3} \mathrm{Ac}\right), 55.4\left(\mathrm{CH}_{3} \mathrm{OMe}\right), 67.8$ (C-6), 68.3 (C-4), 68.6 (C-5), 70.4 (C-3), 70.7 (C2), $71.6\left(\mathrm{CH}_{2} \mathrm{Bn}\right), 72.0$ (C-4’), 74.2 ( $\left.\mathrm{CH}_{2} \mathrm{Bn}\right), 74.3$ (C-2’), 75.5 (C-5’), 80.3 (C-3’), 96.7 (C-1), 98.9 (C-1'), 127.8 - 128.6 (CH Arom), 136.8 ( $\left.\mathrm{C}_{\mathrm{q}} \mathrm{Bn}\right), 137.5$ ( $\left.\mathrm{C}_{\mathrm{q}} \mathrm{Bn}\right), 169.3$ (C=O Ac or lactone), 170.1 (C=O Ac or lactone), 170.1 ( $\mathrm{C}=\mathrm{O}$ Ac or lactone), 171.7 ( $\mathrm{C}=\mathrm{O}$ Ac or lactone); HRMS: $\left(\mathrm{M}+\mathrm{NH}_{4}\right)$ calcd for $\mathrm{C}_{33} \mathrm{H}_{38} \mathrm{O}_{14} 676.26053$, found 676.26440. 


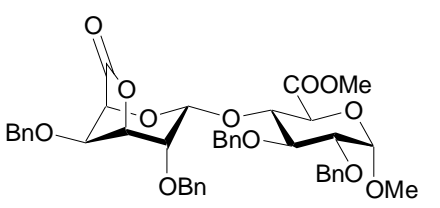

described in the general procedure delivering the title compound $\mathbf{2 3}$ as a colorless oil. TLC: 30\% EtOAc/PE; $[\alpha]_{D}^{22}:+6.0\left(\mathrm{c}=0.5, \mathrm{CHCl}_{3}\right)$; IR $\left(\right.$ neat, $\left.\mathrm{cm}^{-1}\right): 915,1030,1057,1080,1219,1360,1450$, 1504, 1730, 1809, 2925; ${ }^{1} \mathrm{H}$ NMR (400 MHz, $\left.\mathrm{CDCl}_{3}\right) \delta=3.39$ (s, 3H, $\mathrm{CH}_{3} \mathrm{OMe}$ ), 3.53 (dd, $1 \mathrm{H}, J=5.0$ Hz. $J=2.4 \mathrm{~Hz}, \mathrm{H}-2$ '), 3.56 (dd, 1H, $J=6.5 \mathrm{~Hz}, J=3.2 \mathrm{~Hz}, \mathrm{H}-2$ ), 3.67 (s, 3H, $\mathrm{CH}_{3} \mathrm{COOMe}$ ), 3.87 (m, $1 \mathrm{H}, J=2.5 \mathrm{~Hz}, \mathrm{H}-3, \mathrm{H}-5), 4.00$ (s, $1 \mathrm{H}, \mathrm{H}-4^{\prime}$ ), 4.15 (dd, $1 \mathrm{H}, J=7.3 \mathrm{~Hz}, J=2.5 \mathrm{~Hz}, \mathrm{H}-4$ ), 4.30 (d, $1 \mathrm{H}, J$ $=11.9 \mathrm{~Hz}, \mathrm{CH} H \mathrm{Bn}), 4.34$ (s, 1H, H-5'), 4.51 (s, 2H, $\left.\mathrm{CH}_{2} \mathrm{Bn}\right), 4.54$ (dd, $1 \mathrm{H}, J=5.0 \mathrm{~Hz}, J=1.6 \mathrm{~Hz}, \mathrm{H}-$ 3’), 4.59 (d, 1H, $J=11.7 \mathrm{~Hz}, \mathrm{CH} H \mathrm{Bn}$ ), 4.60 (s, 1H, H-1), 4.61 (d, 1H, $J=12.0 \mathrm{~Hz}, \mathrm{CHHBn}$ ), 4.72 (d, 1H, $J=11.9 \mathrm{~Hz}, \mathrm{CHHBn}), 4.75$ (d, 1H, $J=12.0 \mathrm{~Hz}, \mathrm{CHHBn}), 4.99$ (d, 1H, $J=11.7 \mathrm{~Hz}, \mathrm{CHHBn}), 5.03$ (d, $\left.1 \mathrm{H}, J=2.4 \mathrm{~Hz}, \mathrm{H}-1^{\prime}\right), 7.18-7.35$ (m, 20H, CH Arom); ${ }^{13} \mathrm{C}$ NMR $\left(100 \mathrm{MHz}, \mathrm{CDCl}_{3}\right) \delta=52.3\left(\mathrm{CH}_{3}\right.$ COOMe), 55.8 ( $\mathrm{CH}_{3} \mathrm{OMe}$ ), 70.3 (C-4'), 71.3 ( $\left.\mathrm{CH}_{2} \mathrm{Bn}\right), 71.9$ (C-4), 74.2 ( $\left.\mathrm{CH}_{2} \mathrm{Bn}\right), 74.3$ (C-2'), 74.4 ( $\mathrm{CH}_{2} \mathrm{Bn}$ ), 74.5 ( $\mathrm{CH}_{2} \mathrm{Bn}$ ), 75.4 (C-5’), 78.2 (C-5 or C-3), 79.2 (C-2), 80.4 (C-3 or C-5), 98.4 (C-1), 99.1 (C-1'), 127.3 - 128.6 (CH Arom), 137.6 ( $\left.\mathrm{C}_{\mathrm{q}} \mathrm{Bn}\right), 138.6$ ( $\left.\mathrm{C}_{\mathrm{q}} \mathrm{Bn}\right), 168.9$ (C=O COOMe), 172.6 (C=O lactone); HRMS: $\left(\mathrm{M}+\mathrm{NH}_{4}\right)$ calcd for $\mathrm{C}_{48} \mathrm{H}_{48} \mathrm{O}_{13}$ 850.34387, found 850.34180.

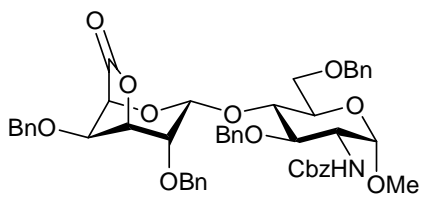

Methyl (2-(N-benzyloxycarbonyl)-amino-3,6-di-O-benzyl-2-deoxy-4(2,4-di-O-benzyl- $\alpha$-D-galactopyranosidurono-6,3-lactone) $\quad$ - $\alpha$-Dglucopyranoside (25): Donor $\mathbf{7}$ was glycosidated with acceptor 24 in the same way as described in the general procedure delivering the title compound $\mathbf{2 5}$ as a colorless oil. N.B.: The amount of TTBP added to the reaction mixture was 1 equiv. with respect to the amount of Tf 2 O. TLC: $30 \%$ EtOAc/PE; $[\alpha]_{\mathrm{D}}^{22}:+31.4\left(\mathrm{c}=1, \mathrm{CHCl}_{3}\right)$; IR (neat, $\left.\mathrm{cm}^{-1}\right): 912,1028,1102,1153$, 1211, 1356, 1401, 1724, 1753; ${ }^{1} \mathrm{H}$ NMR (400 MHz, $\left.\mathrm{CDCl}_{3}\right) \delta=3.36$ (s, 3H, $\mathrm{CH}_{3} \mathrm{OMe}$ ), 3.47 (d, $1 \mathrm{H}, J$ = $\left.2.8 \mathrm{~Hz}, \mathrm{H}-2^{\prime}\right), 3.61$ (t, 1H, $\left.J=8.8 \mathrm{~Hz}, \mathrm{H}-3\right), 3.63-3.75$ (m, 2H, H-5 and H-6), 3.78 (t, 1H, $J=8.8$ Hz, H-4), 3.89 (d, 1H, $J=10.8$ Hz. H-6), 3.99 (s, 1H, H-4'), 4.12 (t, 1H, $J=10.0$ Hz, $J=8.0$ Hz, H-2), 4.28 (s, 1H, H-5'), 4.37 (d, 1H, J = 12.0 Hz, CHHBn), 4.44 (d, 1H, J = 11.6 Hz, CHHBn), 4.49 (s, 1H, H-3'), 4.51 (d, 1H, $J=10.8 \mathrm{~Hz}, \mathrm{CHHBn}$ ), 4.60 (d, 1H, $J=11.6 \mathrm{~Hz}, \mathrm{CHHBn}$ ), 4.65 (d, 1H, $J=12.0 \mathrm{~Hz}$, CHHBn), 4.67 (s, 1H, H-1), 4.72 (d, 1H, $J=10.8 \mathrm{~Hz}, \mathrm{CHHBn}), 5.00$ (d, 1H, $J=10.0 \mathrm{~Hz}, \mathrm{NH}$ ), 5.07 (d, 1H, $J=10.0 \mathrm{~Hz}, \mathrm{CH} H \mathrm{Bn}$ ), 5.10 (s, 1H, H-1'), 5.11 (d, 1H, $J=10.0 \mathrm{~Hz}, \mathrm{CHHBn}), 7.16-7.65$ (m, 25H, 
CH Arom); ${ }^{13} \mathrm{C}$ NMR (100 MHz, $\left.\mathrm{CDCl}_{3}\right) \delta=54.6$ (C-2), 55.0 ( $\left.\mathrm{CH}_{3} \mathrm{OMe}\right), 66.8$ (C-6’), 69.9 (C-4), 71.2 ( $\left.\mathrm{CH}_{2} \mathrm{Cbz}\right), 71.8$ (C-4’), 73.4 ( $\left.\mathrm{CH}_{2} \mathrm{Bn}\right), 74.4$ ( $\left.\mathrm{CH}_{2} \mathrm{Bn}\right), 74.8$ (C-5’), 75.6 (C-2’), 79.6 (C-3’), 81.4 (C-3), 98.9 (C-1), 101.3 (C-1'), 126.8 - 130.9 (CH Arom), 136.7 (C $\left.\mathrm{C}_{\mathrm{q}} \mathrm{Bn}\right), 137.7$ (C $\left.\mathrm{C}_{\mathrm{q}} \mathrm{Bn}\right), 137.8$ (C Bn), $138.3\left(\mathrm{C}_{\mathrm{q}} \mathrm{Bn}\right), 155.6(\mathrm{C}=\mathrm{O} \mathrm{Cbz}), 171.1\left(\mathrm{C}=\mathrm{O}\right.$ lactone); HRMS: $(\mathrm{M}+\mathrm{Na})$ calcd for $\mathrm{C}_{49} \mathrm{H}_{51} \mathrm{NO}_{12}$ 868.33035, found 868.33606.

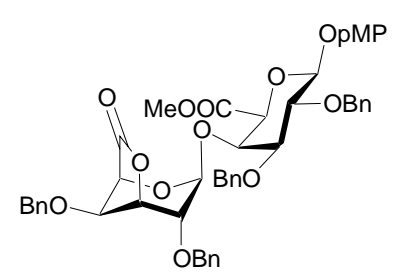

Methyl (para-methoxyphenyl 2,3-di-O-benzyl-4-(2,4-di-O-benzyl- $\beta$-Dgalactopyranosidurono-6,3-lactone)- $\alpha$-D-galactopyranoside) uronate (27 $)$ : Donor 7 was glycosidated with acceptor 26 in the same way as described in the general procedure delivering title compound $27 \alpha / \beta$ as a colorless oil. The anomers were nicely separated with flash chromatography. TLC: 30\% EtOAc/PE; $[\alpha]_{\mathrm{D}}^{22}:-30.0\left(\mathrm{c}=1, \mathrm{CHCl}_{3}\right)$; IR (neat, $\left.\mathrm{cm}^{-1}\right): 910,1028,1058,1080,1217,1363,1454,1506,1735$, 1809, 2922; ${ }^{1} \mathrm{H}$ NMR (400 MHz, $\left.\mathrm{CDCl}_{3}\right) \delta=3.58$ (dd, $1 \mathrm{H}, J=8.8 \mathrm{~Hz}, J=4.6 \mathrm{~Hz}, J=3.2 \mathrm{~Hz}, \mathrm{H}-3$ ), 3.74 (s, 3H, $\mathrm{CH}_{3} \mathrm{OMe}$ or COOMe), 3.75 (s, 3H, $\mathrm{CH}_{3} \mathrm{COOMe}$ or OMe), 3.91 (dd, $1 \mathrm{H}, J=8.8 \mathrm{~Hz}, J=$ 1.6 Hz, H-2), 3.93 (s, 1H, H-2'), 4.05 (s, 1H, H-5), 4.12 (s, 1H, H-4'), 4.50 (d, 1H, J = $11.6 \mathrm{~Hz}$, CHHBn), 4.54 (s, 1H, H-4), 4.56 (d, 1H, $J=11.6$ Hz, CHHBn), 4.58 (s, 1H, H-5'), 4.62 (d, 1H, $J=$ $12.0 \mathrm{~Hz}, \mathrm{CH} H \mathrm{Bn}$ ), 4.67 (d, 1H, $J=11.6 \mathrm{~Hz}, \mathrm{CHHBn}), 4.70$ (s, 1H, H-3'), 4.78 (d, 1H, $J=10.8 \mathrm{~Hz}$, CHHBn), 4.80 (s, 1H, H-1'), 4.81 (d, 1H, $J=8.8 \mathrm{~Hz}, \mathrm{H}-1$ ), 4.85 (d, 1H, $J=12.0 \mathrm{~Hz}, \mathrm{CHHBn}$ ), 4.86 (d, $1 \mathrm{H}, J=11.6 \mathrm{~Hz}, \mathrm{CHHBn}), 5.02$ (d, 1H, $J=10.8 \mathrm{~Hz}, \mathrm{CHHBn}), 6.78$ (d, 2H, $J=9.0 \mathrm{~Hz}, 2 \mathrm{xCH} \mathrm{pMP}$ ), 7.03 (d, 2H, $J=9.0 \mathrm{~Hz}, 2 \mathrm{xCH}$ pMP), $7.23-7.42$ (CH Arom); ${ }^{13} \mathrm{C}$ NMR $\left(100 \mathrm{MHz}, \mathrm{CDCl}_{3}\right) \delta=51.7$ ( $\mathrm{CH}_{3} \mathrm{OMe}$ or COOMe), 53.9 ( $\mathrm{CH}_{3} \mathrm{COOMe}$ or $\left.\mathrm{OMe}\right), 69.6\left(\mathrm{CH}_{2} \mathrm{Bn}\right), 71.3$ (C-4), 71.6 (C-5), 74.2 ( $\left.\mathrm{CH}_{2} \mathrm{Bn}\right), 74.6$ (C-2’), 75.6 ( $\left.\mathrm{CH}_{2} \mathrm{Bn}\right), 75.7$ (C-5’), 75.8 (C-4), 78.0 (C-2), 78.8 (C-3), 80.4 (C-3’), 99.1 (C-1'), 102.5 (C-1), 107.4 (CH pMP), 119.6 (CH pMP), 123.8 - 124.4 (CH Arom), 137.8 (C Bn), $137.9\left(\mathrm{C}_{\mathrm{q}} \mathrm{Bn}\right), 138.4\left(\mathrm{C}_{\mathrm{q}} \mathrm{Bn}\right), 138.8\left(\mathrm{C}_{\mathrm{q}} \mathrm{Bn}\right), 151.7$ ( $\left.\mathrm{C}_{\mathrm{q}} \mathrm{pMP}\right), 155.6$ ( $\left.\mathrm{C}_{\mathrm{q}} \mathrm{pMP}\right), 167.9$ (C=O lactone), 173.7 (C=O COOMe); HRMS: $\left(\mathrm{M}+\mathrm{NH}_{4}\right)$ calcd for $\mathrm{C}_{48} \mathrm{H}_{48} \mathrm{O}_{13}$ 850.34387, found 850.34180.

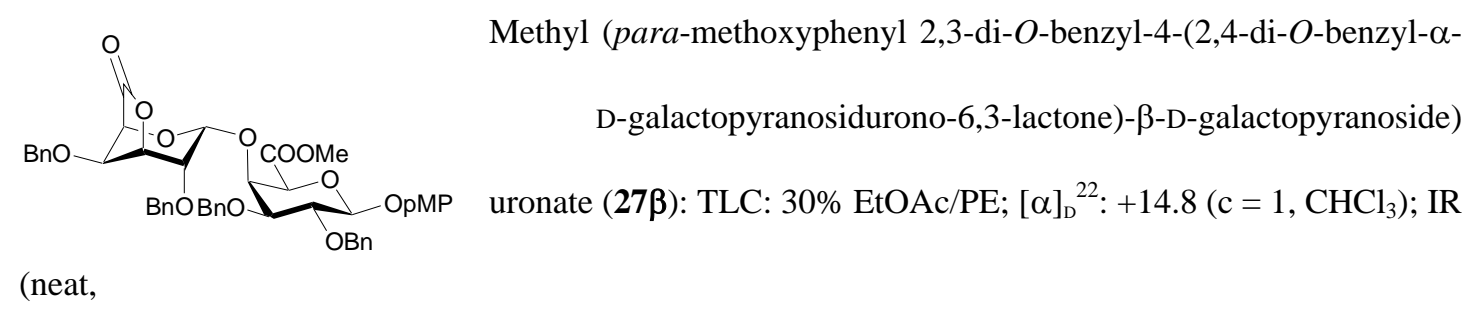


$\left.\mathrm{Cm}^{-1}\right): 910,1028,1058,1080,1217,1363,1454,1506,1735,1809,2922 ;{ }^{1} \mathrm{H}$ NMR (400 MHz, $\left.\mathrm{CDCl}_{3}\right)$ $\delta=3.67$ (dd, $1 \mathrm{H}, J=9.8 \mathrm{~Hz}, J=2.4 \mathrm{~Hz}, \mathrm{H}-3$ ), 3.82 (s, 3H, $\mathrm{CH}_{3}$ COOMe or OMe), 3.97 (s, 3H, $\mathrm{CH}_{3}$ OMe or COOMe), 3.98 (dd, 1H, $J=7.6 \mathrm{~Hz}, J=2.4 \mathrm{~Hz}, \mathrm{H}-2$ ), 4.04 (s, 1H, H-4'), 4.08 (s, 1H, H-5'), 4.11 (d, 1H, $J=4.8 \mathrm{~Hz}, \mathrm{H}-2$ ') 4.23 (d, 1H, $J=12.0 \mathrm{~Hz}, \mathrm{CH} H \mathrm{Bn}$ ), 4.34 (s, 1H, H-5'), 4.39 (d, 1H, $J=$ $12.0 \mathrm{~Hz}, \mathrm{CHHBn}$ ), 4.61 (s, 2H, CH ${ }_{2} \mathrm{Bn}$ ), 4.66 (s, 1H, H-4), 4.68 (d, 1H, J = 4.8 Hz, H-3'), 4.81 (s, 2H, $\mathrm{CH}_{2} \mathrm{Bn}$ ), 4.86 (d, 1H, $J=7.6 \mathrm{~Hz}, \mathrm{H}-1$ ), 4.90 (d, $1 \mathrm{H}, J=10.8 \mathrm{~Hz}, \mathrm{CH} H \mathrm{Bn}$ ), 5.07 (d, $1 \mathrm{H}, J=10.8 \mathrm{~Hz}$, CHHBn), 5.60 (s, 1H, H-1'), 6.87 (d, 2H, $J=9.2 \mathrm{~Hz}, 2 \mathrm{xCH}$ pMP), 7.15 (d, 2H, $J=9.2 \mathrm{~Hz}, 2 \mathrm{xCH}$ pMP), $7.30-7.43$ (CH Arom); ${ }^{13} \mathrm{C}$ NMR (100 MHz, $\left.\mathrm{CDCl}_{3}\right) \delta=52.9\left(\mathrm{CH}_{3}\right.$ OMe or COOMe), 55.6 ( $\mathrm{CH}_{3} \mathrm{COOMe}$ or OMe), 70.7 (C-4'), $71.2\left(\mathrm{CH}_{2} \mathrm{Bn}\right), 72.2\left(\mathrm{CH}_{2} \mathrm{Bn}\right), 73.3$ (C-5), 73.5 (C-4), $74.0\left(\mathrm{CH}_{2}\right.$ Bn), 75.1 ( $\mathrm{CH}_{2} \mathrm{Bn}$ ), 75.9 (C-5’), 77.5 (C-2), 78.1 (C-2’), 78.5 (C-3’), 81.5 (C-3), 100.5 (C-1'), 103.5 (C-1), 114.4 (CH pMP), 119.08 (CH pMP), 127.6 - 128.6 (CH Arom), 136.8 ( $\left.\mathrm{C}_{\mathrm{q}} \mathrm{Bn}\right), 137.1\left(\mathrm{C}_{\mathrm{q}} \mathrm{Bn}\right)$, $137.3\left(\mathrm{C}_{q} \mathrm{Bn}\right), 138.1\left(\mathrm{C}_{\mathrm{q}} \mathrm{Bn}\right), 151.4\left(\mathrm{C}_{\mathrm{q}} \mathrm{pMP}\right), 155.5\left(\mathrm{C}_{\mathrm{q}} \mathrm{pMP}\right), 166.9$ (C=O lactone), $172.0(\mathrm{C}=\mathrm{O}$ COOMe); HRMS: $\left(\mathrm{M}+\mathrm{NH}_{4}\right)$ calcd for $\mathrm{C}_{48} \mathrm{H}_{48} \mathrm{O}_{13}$ 850.34387, found 850.33893.

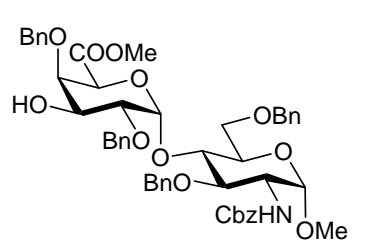

Methyl (2-(N-benzyloxycarbonyl)-amino-3,6-di-O-benzyl-2-deoxy-4(methyl 2,4-di-O-benzyl- $\alpha$-D-galactopyranosyl uronate) - $\alpha$-Dglucopyranoside (28): Lactone 25 was dissolved in anhydrous methanol before a catalytic amount KOtBu (10mol\%) was added. Stirring was continued for 4 h before the mixture was neutralized with Amberlite IR-120 $\mathrm{H}^{+}$-resin. Filtering of the mixture and purification by flash chromatography afforded the title compound $\mathbf{2 8}$ as a colorless oil. TLC: $30 \%$ EtOAc/PE; $[\alpha]_{\mathrm{D}}{ }^{22}$ : +93.6 (c = 1, $\mathrm{CHCl}_{3}$ ); IR (neat, $\mathrm{cm}^{-1}$ ): 912, 1028, 1102, 1153, 1211, 1356, 1401, 1724, 1753; ${ }^{1} \mathrm{H}$ NMR (400 MHz, $\left.\mathrm{CDCl}_{3}\right) \delta=3.33$ (s, 3H, $\mathrm{CH}_{3} \mathrm{OMe}$ ), 3.43 (s, 3H, $\mathrm{CH}_{3} \mathrm{COOMe}$ ), 3.61 (d, $1 \mathrm{H}, J=9.6 \mathrm{~Hz}, \mathrm{H}-$ 6), $3.77-3.80$ (m, 3H, H-4, H-5 and H-2'), 3.85 (d, 1H, $J=9.6$ Hz, H-6), 4.01 (dd, 1H, $J=10.0 \mathrm{~Hz}, J$ = $\left.3.0 \mathrm{~Hz}, \mathrm{H}-3^{\prime}\right), 4.12-4.16$ (m, 2H, H-2 and H-4'), 4.18 (t, 1H, $J=9.6 \mathrm{~Hz}, \mathrm{H}-3$ ), 4.34 (d, 1H, $J=12.0$ Hz, CHHBn), 4.46 (s, 1H, H-5'), 4.52 (d, 1H, $J=11.6 \mathrm{~Hz}, \mathrm{CHHBn}), 4.55$ (d, $1 \mathrm{H}, J=12.0 \mathrm{~Hz}$, CHHBn), 4.56 (s, 2H, $\mathrm{CH}_{2} \mathrm{Bn}$ ), 4.58 (d, 1H, $\left.J=11.6 \mathrm{~Hz}, \mathrm{CHHBn}\right), 4.67$ (d, 1H, $J=11.2 \mathrm{~Hz}, \mathrm{CHHBn}$ ), 4.70 (s, 1H, H-1), 4.72 (d, 1H, $J=11.2 \mathrm{~Hz}, \mathrm{CHHBn}$ ), 4.94 (d, 1H, $J=10.0 \mathrm{~Hz}, \mathrm{NH}), 4.98$ (d, 1H, $J=$ 12.4 Hz, CHHBn), 5.03 (d, 1H, $J=12.4$ Hz, CHHBn), 5.77 (d, 1H, $J=3.2 \mathrm{~Hz}, \mathrm{H}-1$ '), 7.18 - 7.34 (m, 25H, CH Arom); ${ }^{13} \mathrm{C}$ NMR (100 MHz, $\left.\mathrm{CDCl}_{3}\right) \delta=52.0\left(\mathrm{CH}_{3} \mathrm{COOMe}\right), 53.9$ (C-2), $55.1\left(\mathrm{CH}_{3} \mathrm{OMe}\right)$, $66.9\left(\mathrm{CH}_{2} \mathrm{Bn}\right), 68.8$ ( $\left.\mathrm{CH}_{2} \mathrm{Bn}\right), 69.4$ (C-3’), 70.2 (C-4, C-5 or C-2’), 70.9 (C-5’), $72.1\left(\mathrm{CH}_{2} \mathrm{Bn}\right), 72.3$ 
(C-4'), $72.8\left(\mathrm{CH}_{2} \mathrm{Bn}\right), 73.3\left(\mathrm{CH}_{2} \mathrm{Bn}\right), 74.9\left(\mathrm{CH}_{2} \mathrm{Bn}\right), 75.6$ (C-4, C-5 or C-2’), 77.8 (C-3), 80.7 (C-4, C-5 or C-2’), 96.6 (C-1'), 98.7 (C-1), 126.8 - 128.4 (CH Arom), 137.5 (C $\left.\mathrm{q}_{\mathrm{q}} \mathrm{Bn}\right), 137.9$ (C $\mathrm{q}$ Bn), 138.0 (C $\left.\mathrm{q}_{\mathrm{q}} \mathrm{Bn}\right), 155.7$ (C=O Cbz), 168.9 (C=O COOMe); HRMS: $(\mathrm{M}+\mathrm{Na})$ calcd for $\mathrm{C}_{50} \mathrm{H}_{55} \mathrm{NO}_{13}$ 900.35656, found 900.35333 .

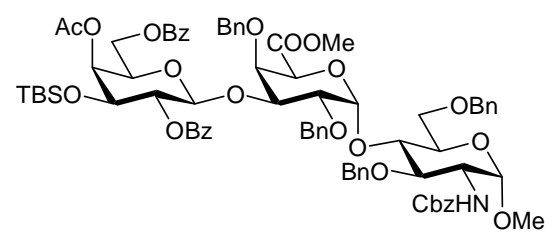

Methyl (2-( $N$-benzyloxycarbonyl)-amino-3.6-di-O-benzyl-2-deoxy-4-O-(methyl 2,4-di-O-benzyl-3-O(4-O-acetyl-2,6-di-O-benzoyl-3-O-tert-butyldimethylsilyl- $\beta$-D-galactopyranosyl)- $\alpha$-D-galactopyranosyl urinate)- $\alpha$-D-glucopyranoside (30): A solution of $63 \mathrm{mg}$ donor 29 (0.1 mmol) and $26 \mathrm{mg}$ diphenylsulfoxide (0.13 mmol, 1.3 equiv.) in DCM (0.05M) was stirred over activated MS3Å for 30 min. The mixture was cooled to $-60^{\circ} \mathrm{C}$ before $21 \mu$ l triflic acid anhydride $(0.13$ mmol, 1.3 equiv.) was added. The mixture was allowed to warm to $-40^{\circ} \mathrm{C}$ in $60 \mathrm{~min}$ followed by addition of $87 \mathrm{mg}$ acceptor 28 (0.1 mmol, 1.0 equiv.) in DCM (0.15M). Stirring was continued and the reaction mixture was allowed to warm to $-15^{\circ} \mathrm{C}$. The reaction mixture was diluted with EtOAc and washed with water. The water/layer was extracted twice with EtOAc. The collected organic layers were dried $\left(\mathrm{MgSO}_{4}\right)$, filtered and concentrated under reduced pressure. Flash chromatography (EtOAc/PE) and removal of the eluent afforded $88 \mathrm{mg}$ of the title compound (0.063 mmol, 63\%) as a light yellow oil. TLC: 30\% EtOAc/PE; $[\alpha]_{\mathrm{D}}^{22}:+34.8\left(\mathrm{c}=1, \mathrm{CHCl}_{3}\right) ;$ IR (neat, $\left.\mathrm{cm}^{-1}\right): 912,1102,1153,1211,1401,1724,1753 ;{ }^{1} \mathrm{H}$ NMR $(600$ MHz, $\left.\mathrm{CDCl}_{3}\right) \delta=-0.13$ (s, 3H, $\mathrm{CH}_{3}, \mathrm{TBDMS}$ ), -0.07 (s, 3H, $\left.\mathrm{CH}_{3}, \mathrm{TBDMS}\right), 0.69$ (s, 9H, C(CH $)_{3}$, TBDMS), 2.19 (s, 3H, $\mathrm{CH}_{3}$ Ac), 3.31 (s, 3H, $\mathrm{CH}_{3} \mathrm{COOMe}$ ), 3.34 (s, 3H, $\mathrm{CH}_{3}, \mathrm{OMe}$ ), 3.51 (d, 1H, $J=$ $10.9 \mathrm{~Hz}, \mathrm{H}-6), 3.60$ (t, 2H, $J=10.2 \mathrm{~Hz}, J=9.3 \mathrm{~Hz}, \mathrm{H}-4$ and H-5), 3.73 (d, 1H, $J=11.0 \mathrm{~Hz}, \mathrm{H}-6), 3.83$ (dd, 1H, $J=8.6 \mathrm{~Hz}, J=3.1 \mathrm{~Hz}, \mathrm{H}-2$ '), 3.91 (m, 2H, H-3'’ and H-5'), 4.02 (t, 1H, $J=9.3 \mathrm{~Hz}, \mathrm{H}-2$ ), 4.07 (t, 1H, $J=9.3 \mathrm{~Hz}, \mathrm{H}-3$ ), 4.10 (d, 1H, $J=12.8 \mathrm{~Hz}, \mathrm{CHHBn}$ ), 4.21 (dd, 1H, $J=10.3 \mathrm{~Hz}, J=2.6 \mathrm{~Hz}$, H-3’), 4.30 (d, 1H, J = $12.8 \mathrm{~Hz}, \mathrm{CHHBn),} 4.32$ (s, 1H, H-4'), 4.36 (s, 1H, H-5'), 4.40 (dd, 1H, $J=11.2$ Hz, $J=5.5 \mathrm{~Hz}, \mathrm{H}-6$ '’), 4.41 (d, 1H, $J=12.1 \mathrm{~Hz}, \mathrm{CHHBn}$ ), 4.43 (d, 1H, $J=12.1 \mathrm{~Hz}, \mathrm{CH} H \mathrm{Bn}), 4.46$ (d, 1H, $J=12.1 \mathrm{~Hz}, \mathrm{CHHBn}), 4.52$ (dd, $1 \mathrm{H}, J=11.2 \mathrm{~Hz}, J=7.0 \mathrm{~Hz}, \mathrm{H}-$ 6’’), 4.63 (d, 1H, $J=11.8 \mathrm{~Hz}, \mathrm{CHHBn}), 4.65$ (d, 1H, $J=12.1 \mathrm{~Hz}, \mathrm{CHHBn}), 4.66$ (s, 1H, H-1), 4.77 (d, 
$1 \mathrm{H}, J=9.9 \mathrm{~Hz}, \mathrm{NH}), 4.89$ (d, 1H, $J=8.0 \mathrm{~Hz}, \mathrm{H}-1$ ') $), 4.95$ (s, 2H, $\mathrm{CH}_{2} \mathrm{Bn}$ ), 4.99 (d, $1 \mathrm{H}, J=11.8 \mathrm{~Hz}$, CHHBn), 4.31 (d, 1H, J = 3.1 Hz, H-1'), 5.40 (s, 1H, H-4'”), 5.54 (t, 1H, $J=8.1 \mathrm{~Hz}, \mathrm{H}-2$ '’), 6.92 8.08 (m, 35H, CH Arom); ${ }^{13} \mathrm{C}$ NMR (150 MHz, $\left.\mathrm{CDCl}_{3}\right) \delta=-4.8$ (2xCH $\mathrm{xHDMS}_{3}$, 17.6 ( $\mathrm{C}_{\mathrm{q}}$ TBDMS), $20.9\left(\mathrm{CH}_{3} \mathrm{Ac}\right), 25.2\left(\mathrm{C}\left(\mathrm{CH}_{3}\right)_{3}\right.$ TBDMS), $51.8\left(\mathrm{CH}_{3} \mathrm{OMe}\right), 54.0$ (C-2), $55.1\left(\mathrm{CH}_{3} \mathrm{COOMe}\right), 62.4$ (C6’'), 66.8 ( $\mathrm{CH}_{2} \mathrm{Cbz}$ ), 68.6 (C-6), 70.2 (C-4’'), 70.5 (C-5), 71.0 (C-3’’ and C-5’'), 71.3 (C-3'), 72.6 (C2’'), 72.8 ( $\left.\mathrm{CH}_{2} \mathrm{Bn}\right), 73.1$ ( $\left.\mathrm{CH}_{2} \mathrm{Bn}\right), 73.5$ ( $\left.\mathrm{CH}_{2} \mathrm{Bn}\right), 74.3$ (C-3), 74.7 ( $\left.\mathrm{CH}_{2} \mathrm{Bn}\right), 75.1$ (C-2'), 75.2 (C-3), 77.8 (C-3’’), 80.0 (C-4), 97.8 (C-1’), 98.6 (C-1), 101.7 (C-1’’), 127.1 - 133.3 (CH Arom), 128.6 (C Bz), $129.5\left(\mathrm{C}_{\mathrm{q}} \mathrm{Bz}\right), 133.2\left(\mathrm{C}_{\mathrm{q}} \mathrm{Bn}\right), 138.2\left(\mathrm{C}_{\mathrm{q}} \mathrm{Bn}\right), 138.4\left(\mathrm{C}_{\mathrm{q}} \mathrm{Bn}\right), 138.5\left(\mathrm{C}_{\mathrm{q}} \mathrm{Bn}\right), 155.7(\mathrm{C}=\mathrm{O} \mathrm{Cbz})$, 165.0 (C=O Bz, Ac or COOMe), 166.0 ( $\mathrm{C}=\mathrm{O} \mathrm{Bz}, \mathrm{Ac}$ or COOMe), $168.6(\mathrm{C}=\mathrm{O} \mathrm{Bz}, \mathrm{Ac}$ or COOMe), $170.2\left(\mathrm{C}=\mathrm{O} \mathrm{Bz}\right.$, Ac or COOMe). HRMS: $\left(\mathrm{M}+\mathrm{Na}\right.$ ) calcd for $\mathrm{C}_{78} \mathrm{H}_{89} \mathrm{NO}_{21} \mathrm{Si} 1426.52575$, found 1426.53050.

(i) Crich, D.; Smith, M.; Yao, Q.; Picione, J. Synthesis, 2001, 323 - 326 


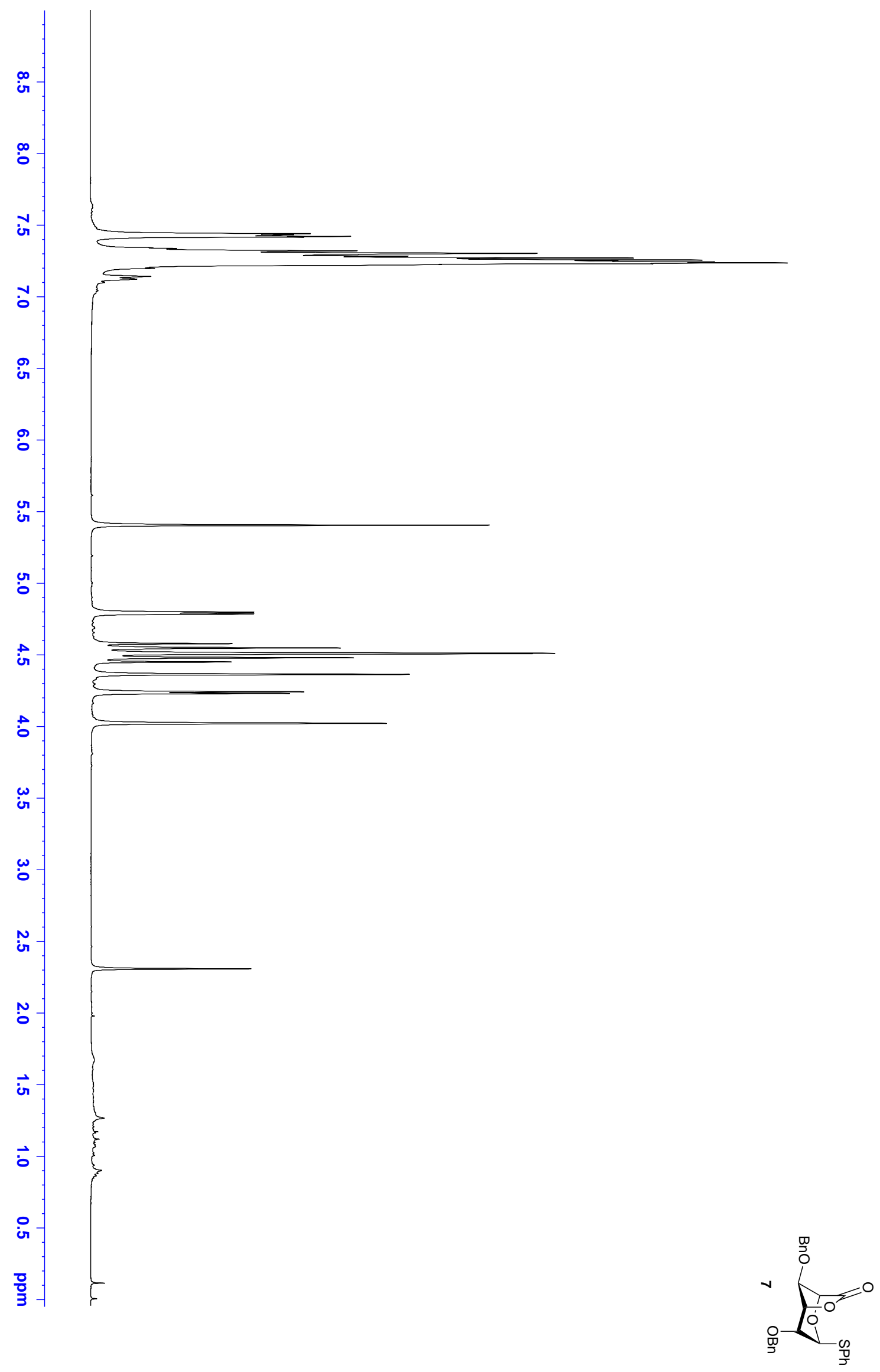

12 of 43 


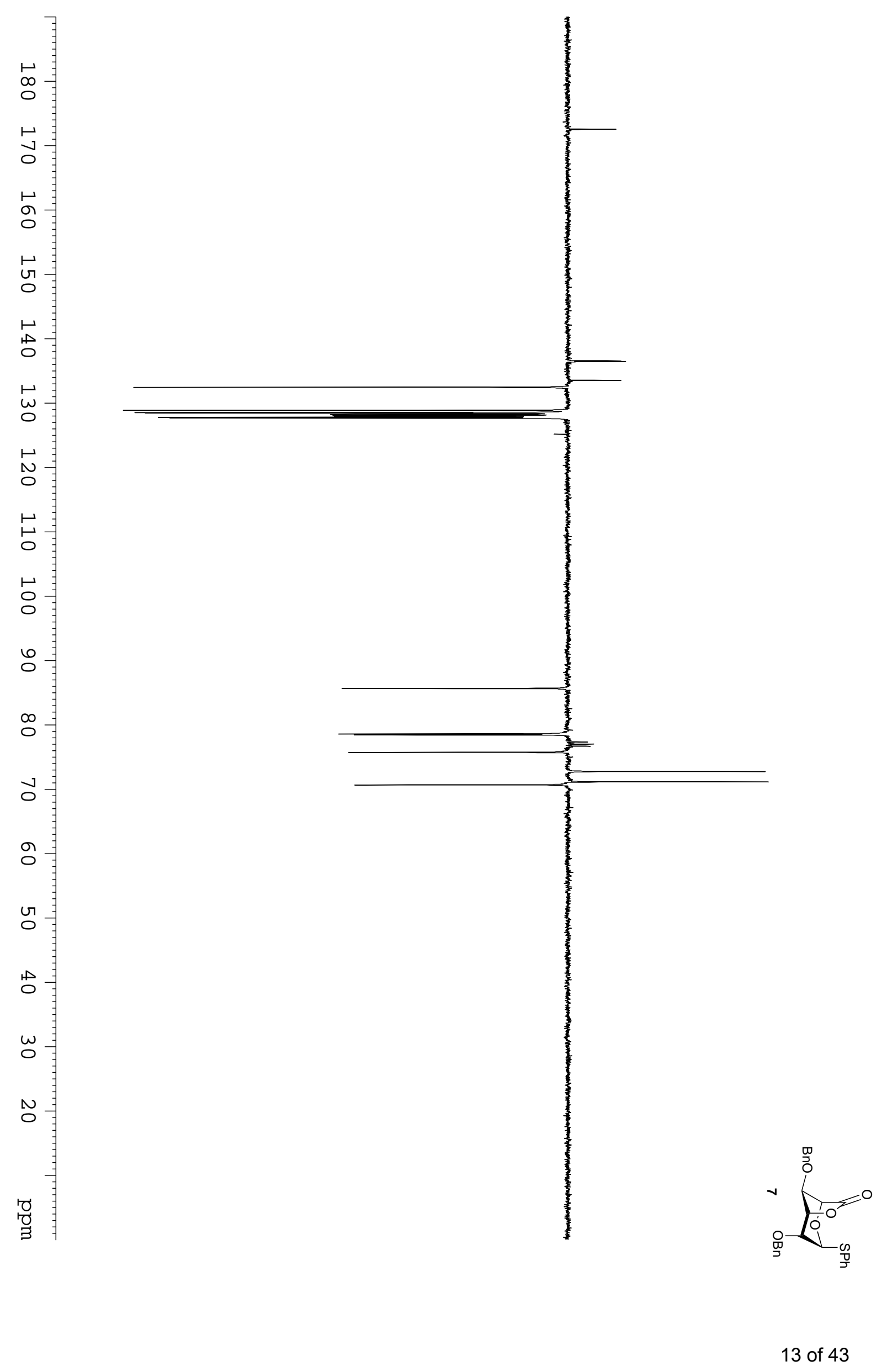




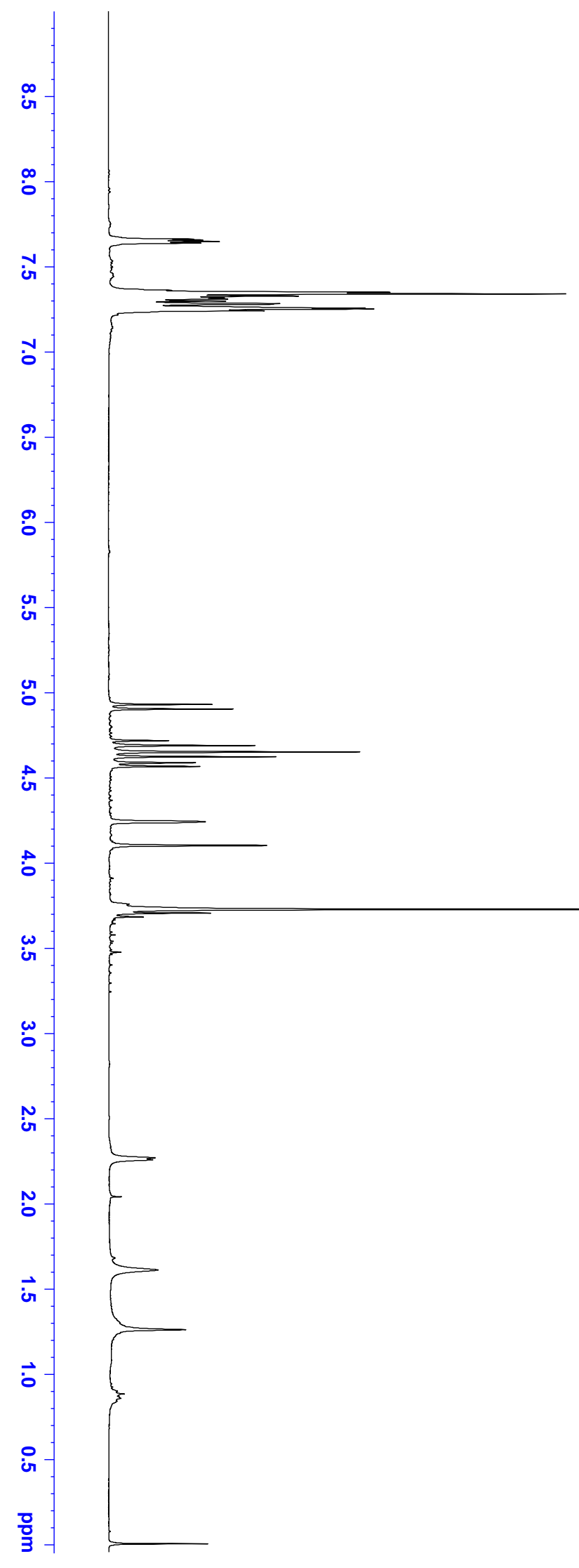

$\infty \int_{\substack{0 \\ \frac{0}{3}}}^{\frac{1}{0}}$

14 of 43 


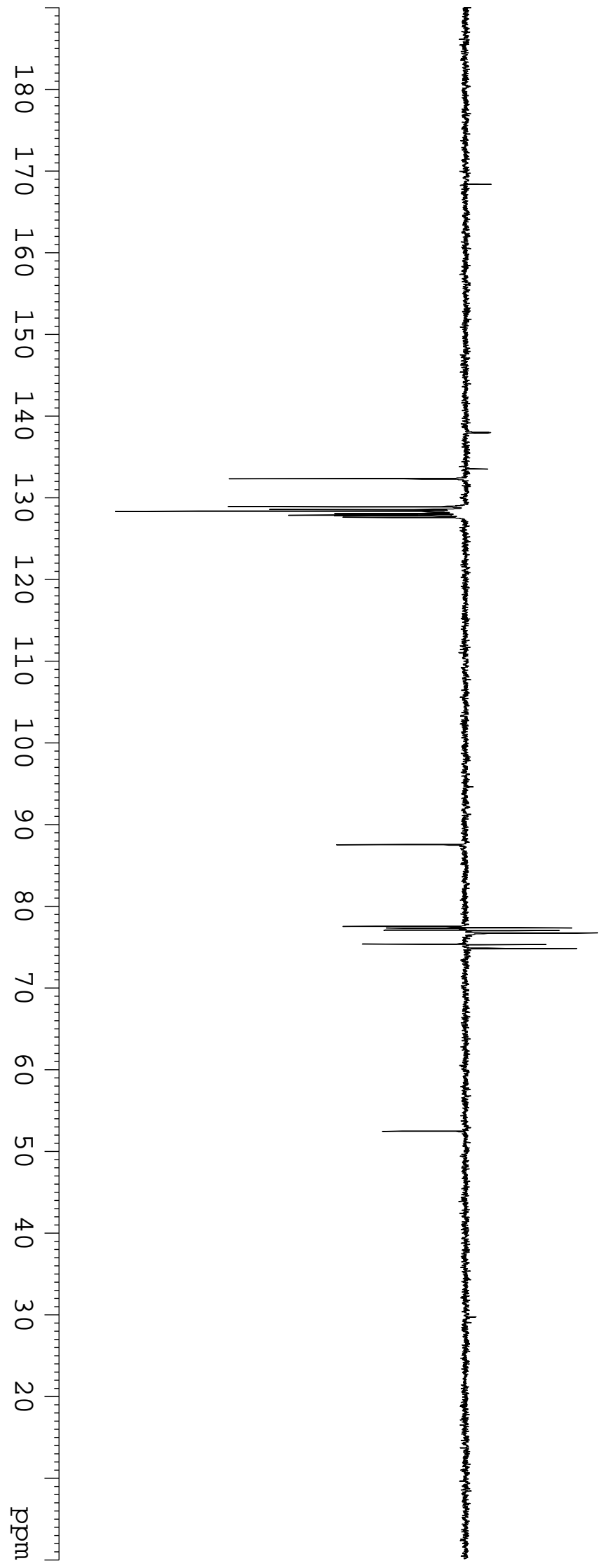

$\infty \underbrace{\frac{1}{0}}_{\substack{0 \\ \frac{0}{3}}}$

15 of 43 

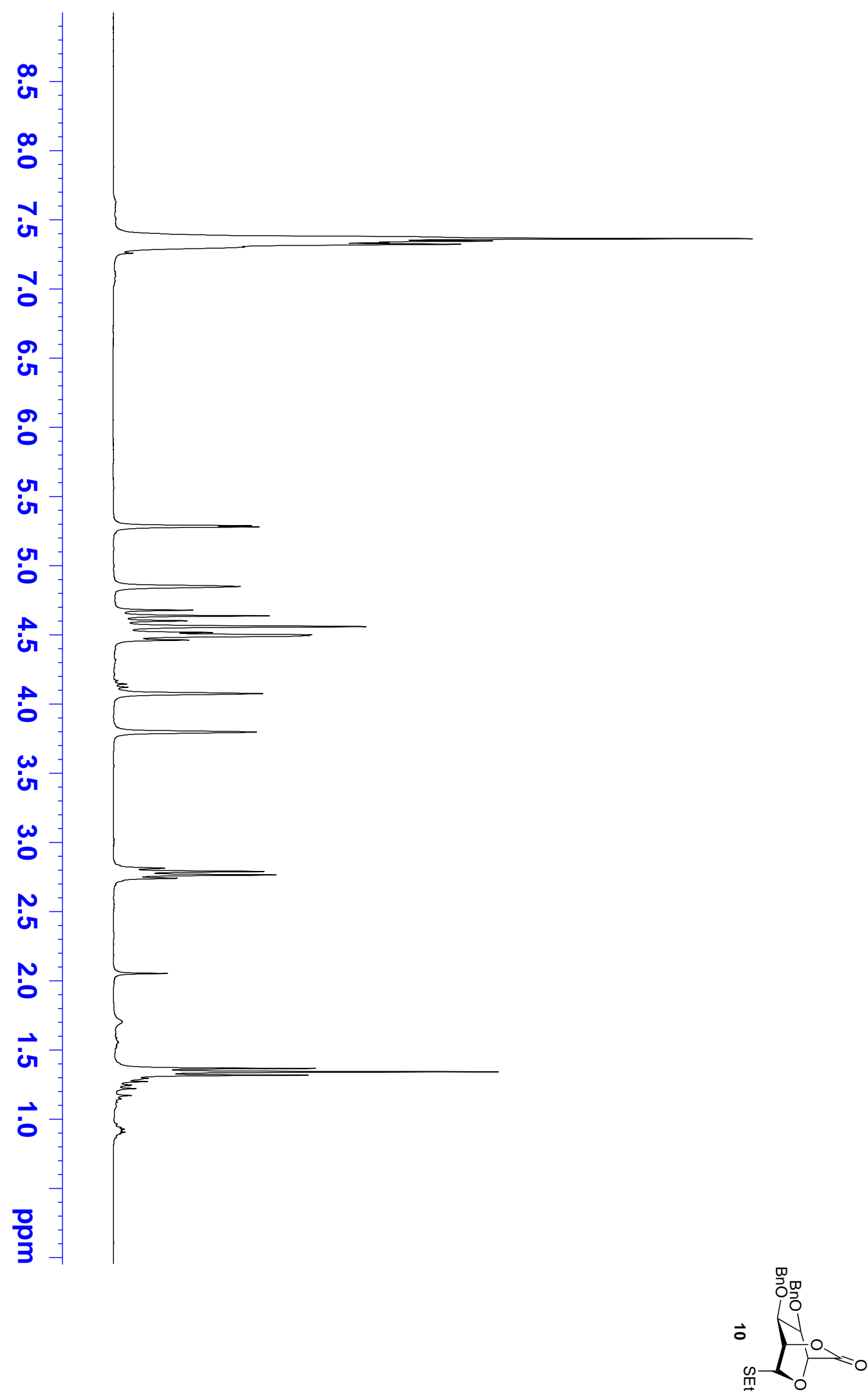

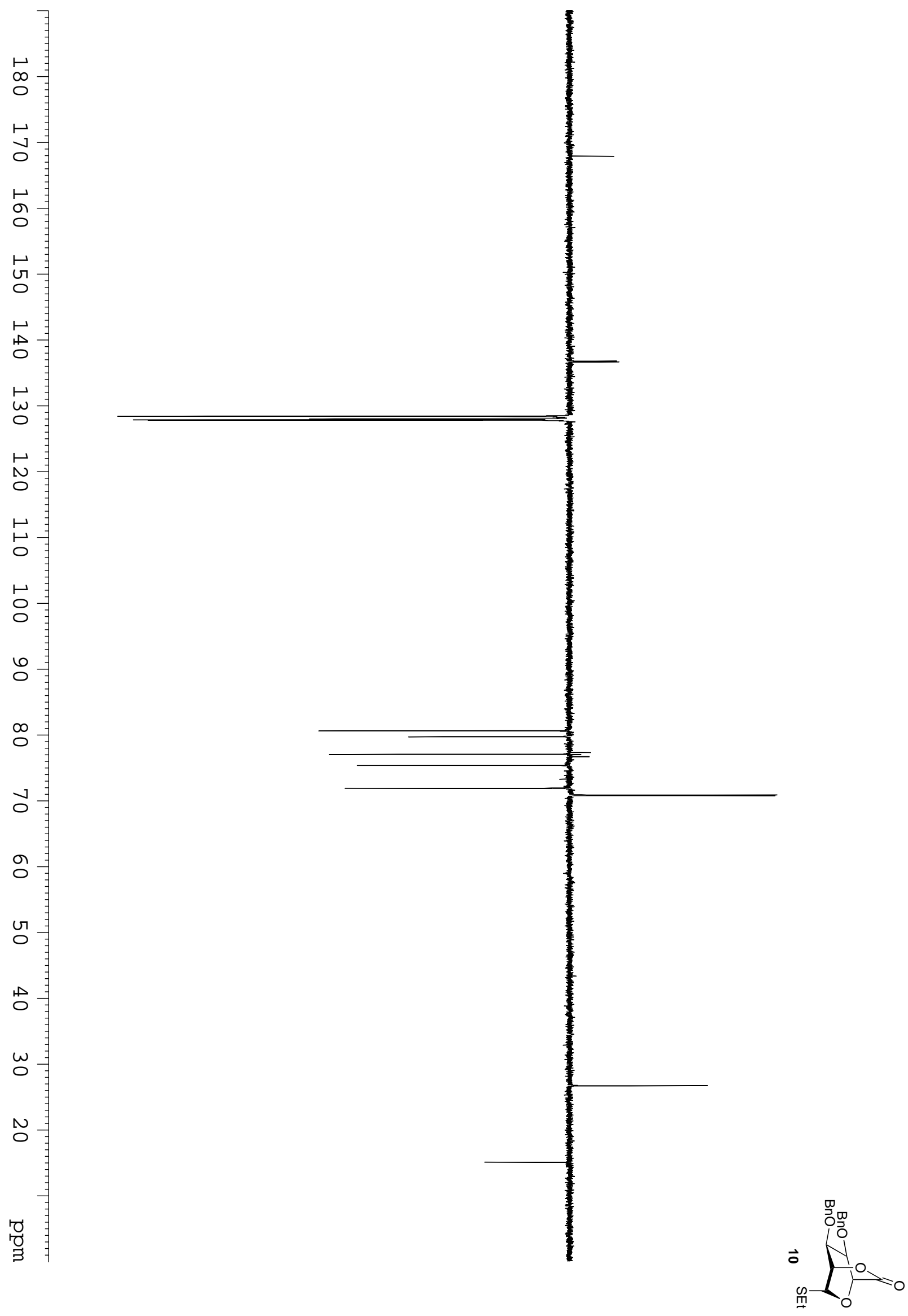

17 of 43 


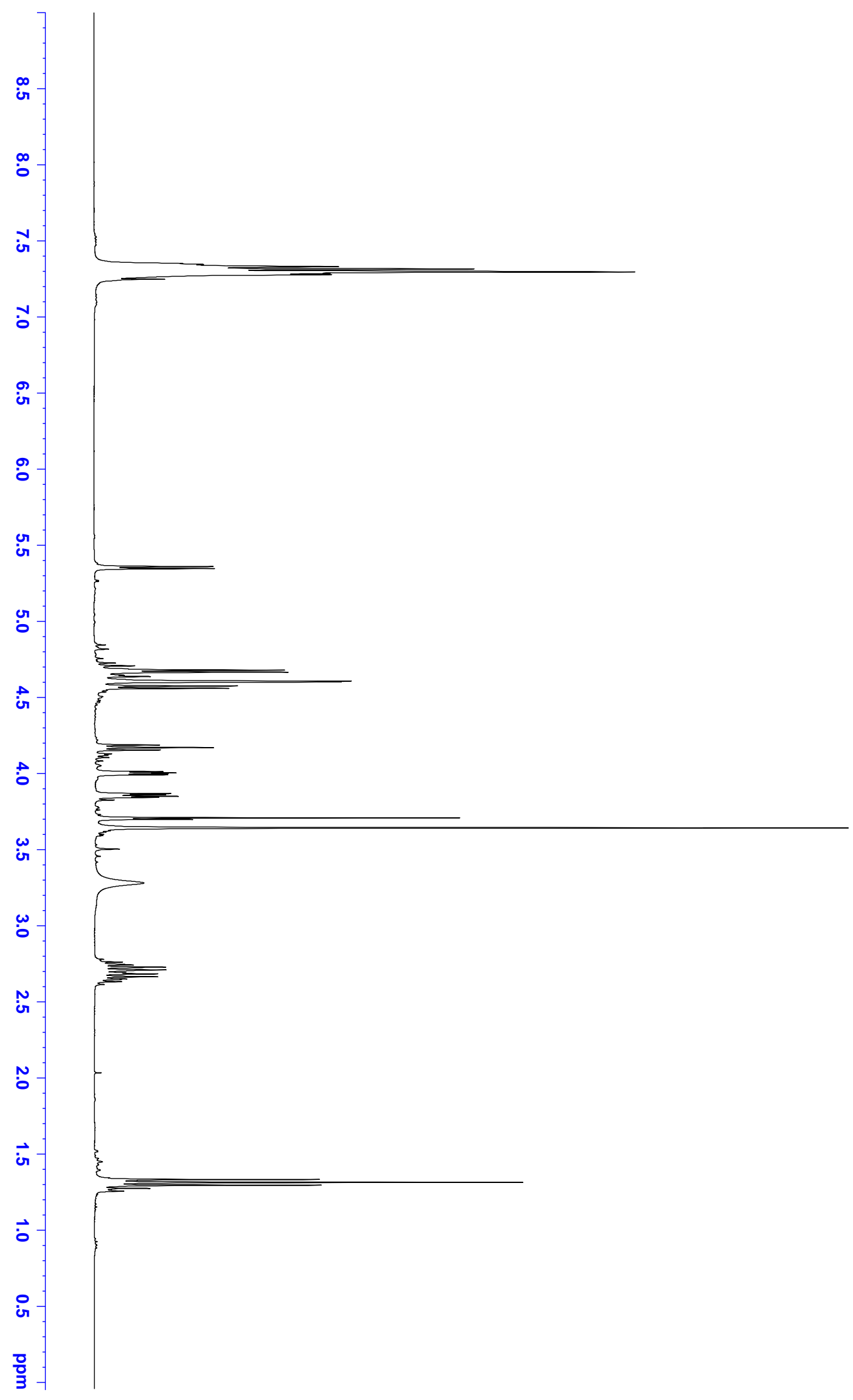



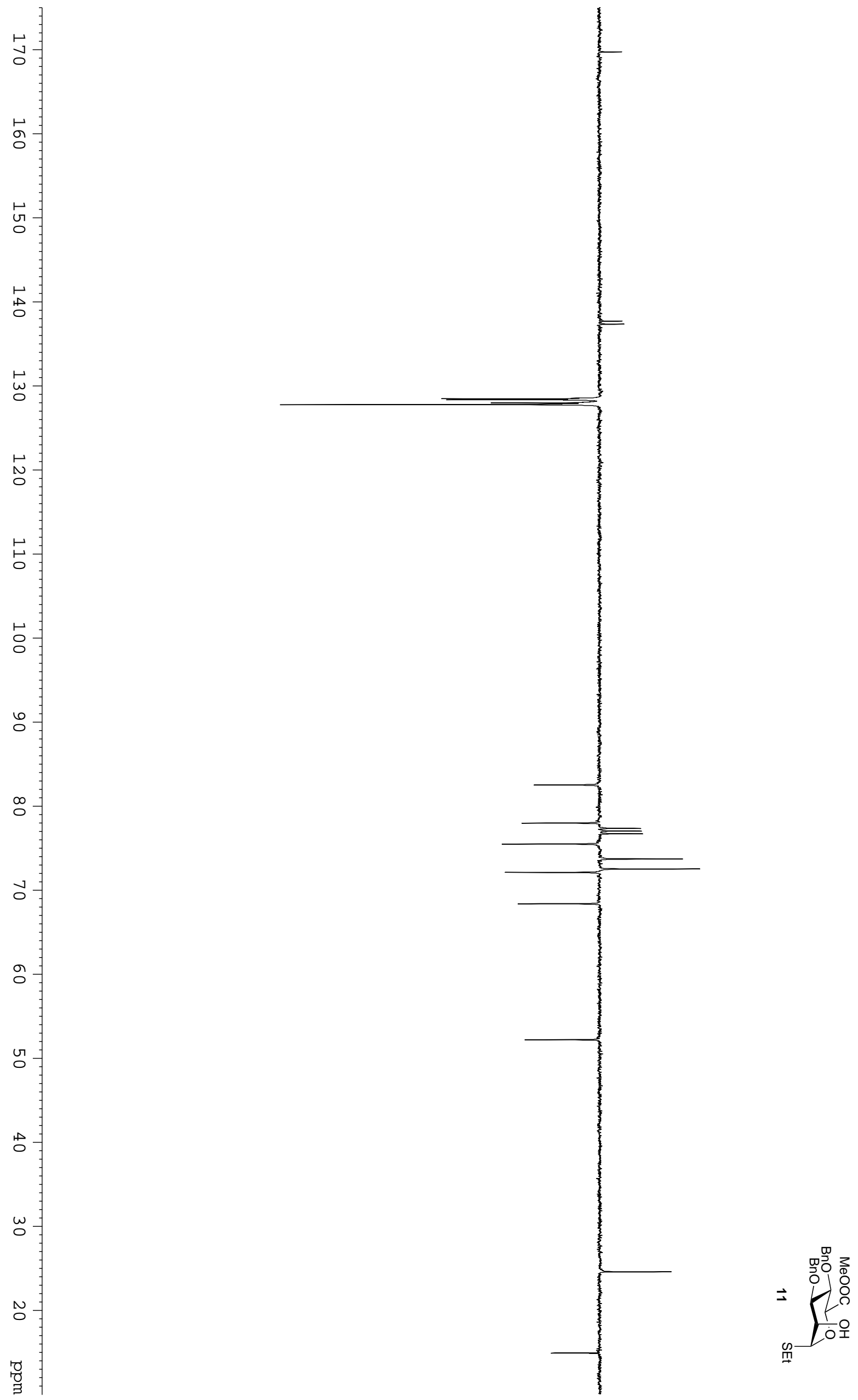

19 of 43 

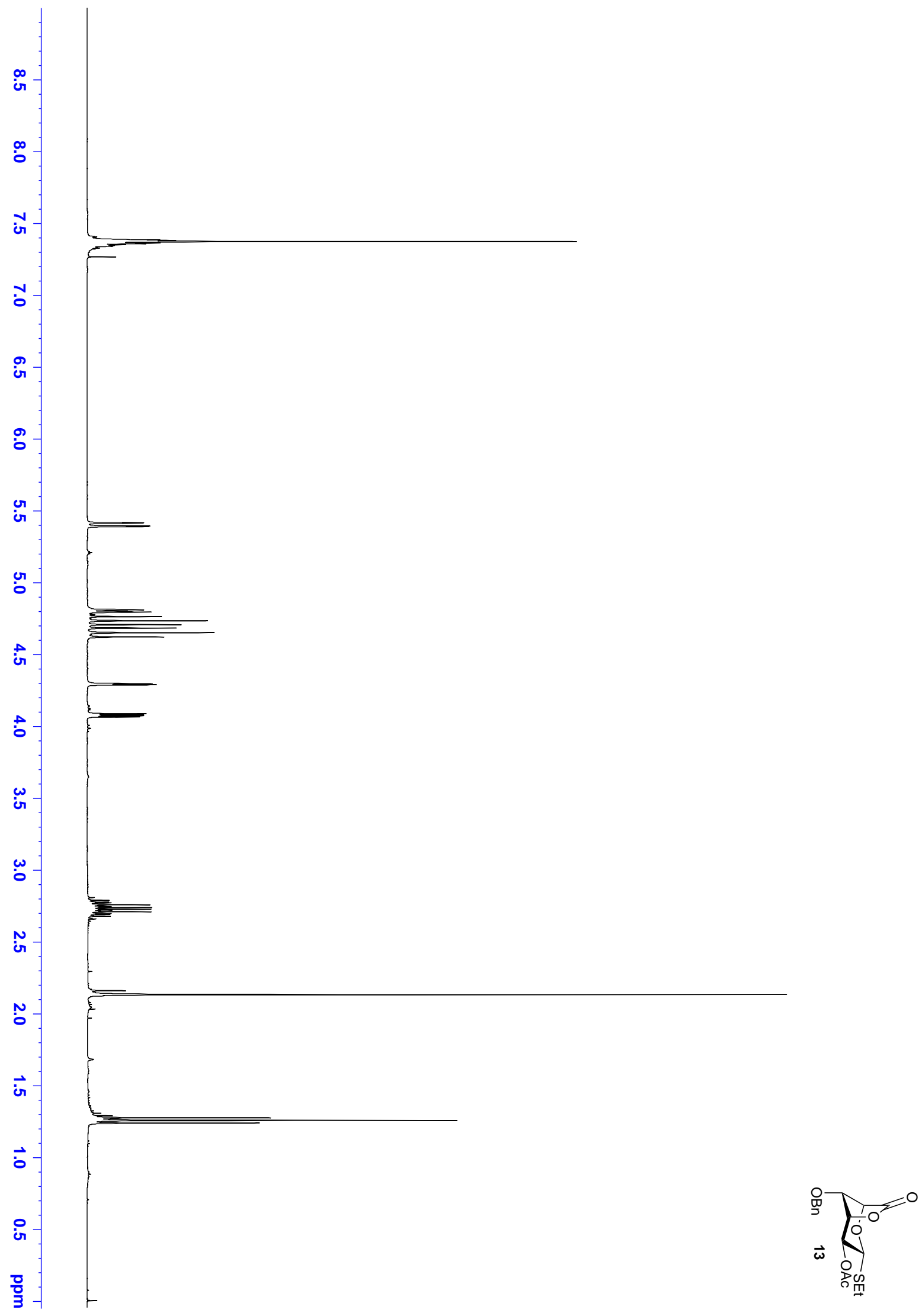

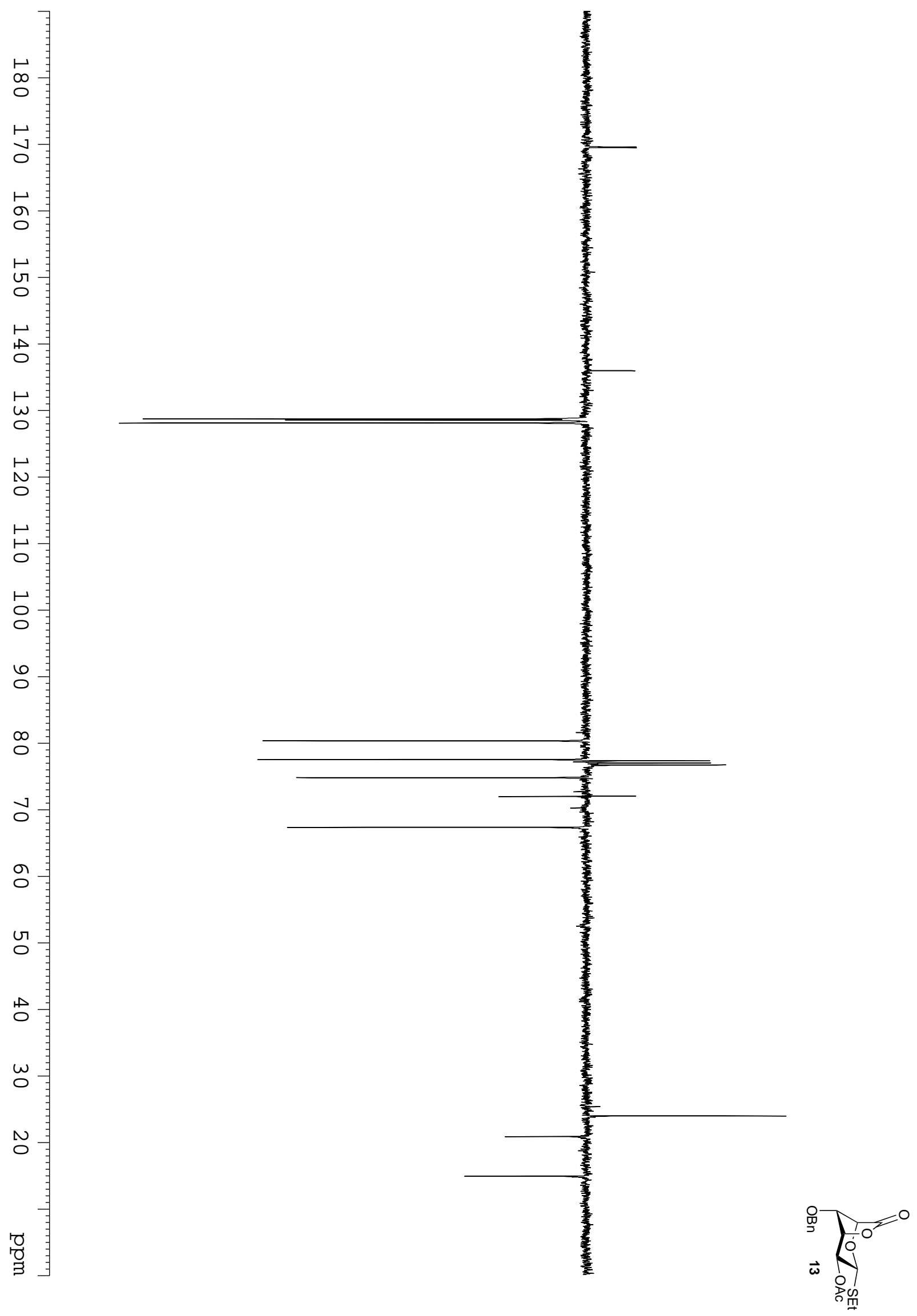

21 of 43 


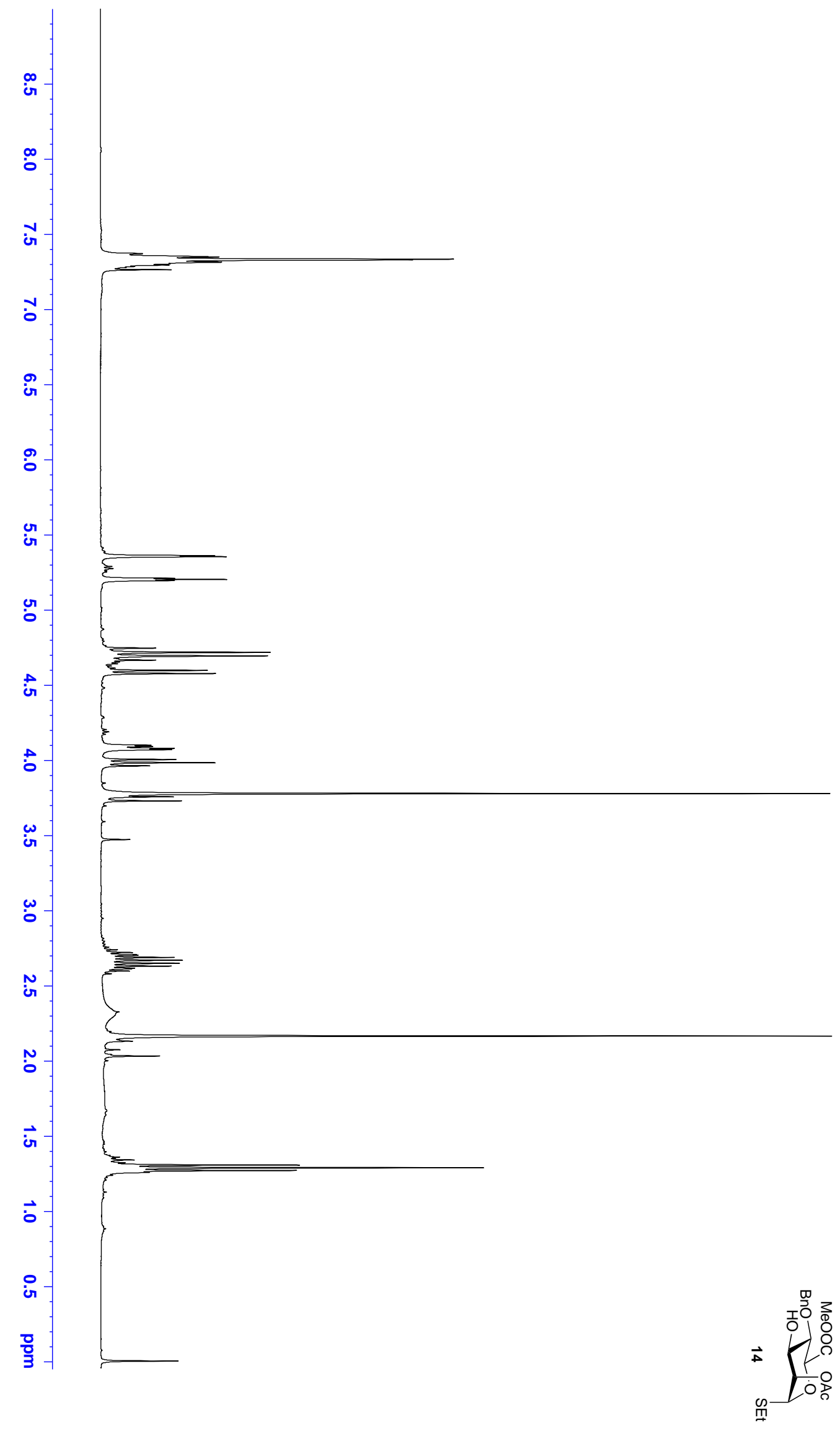




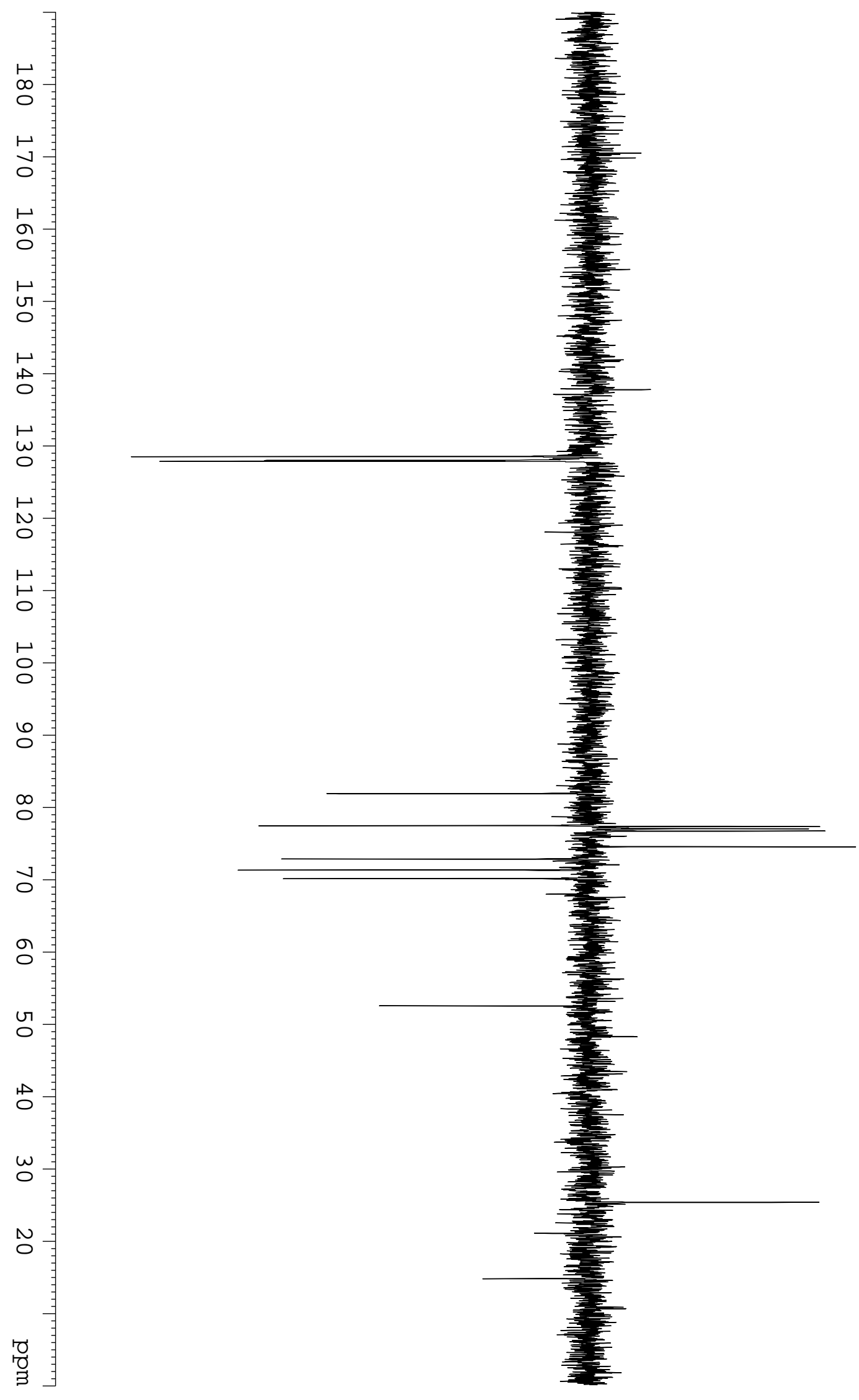

•

23 of 43 


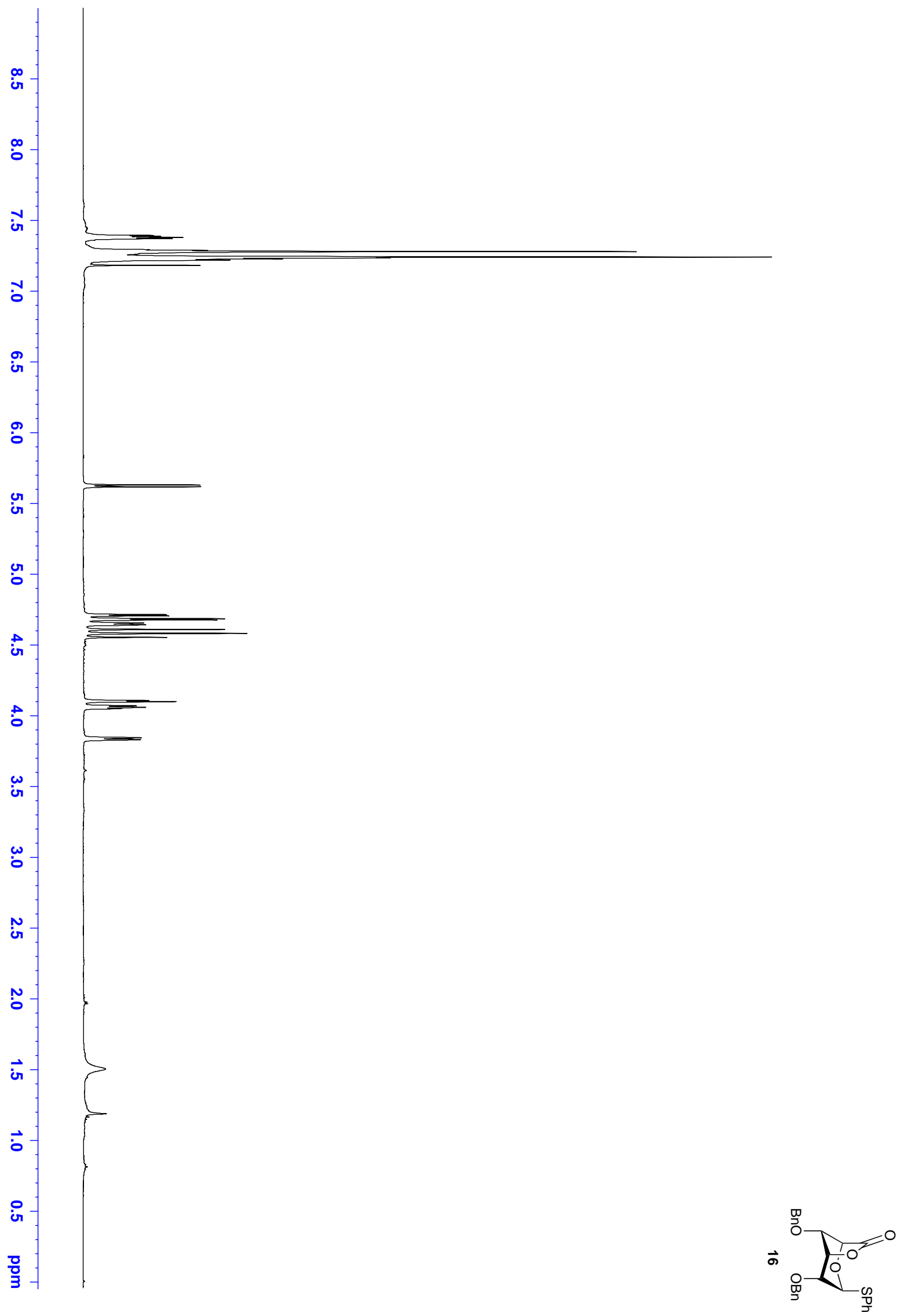




$$
7
$$



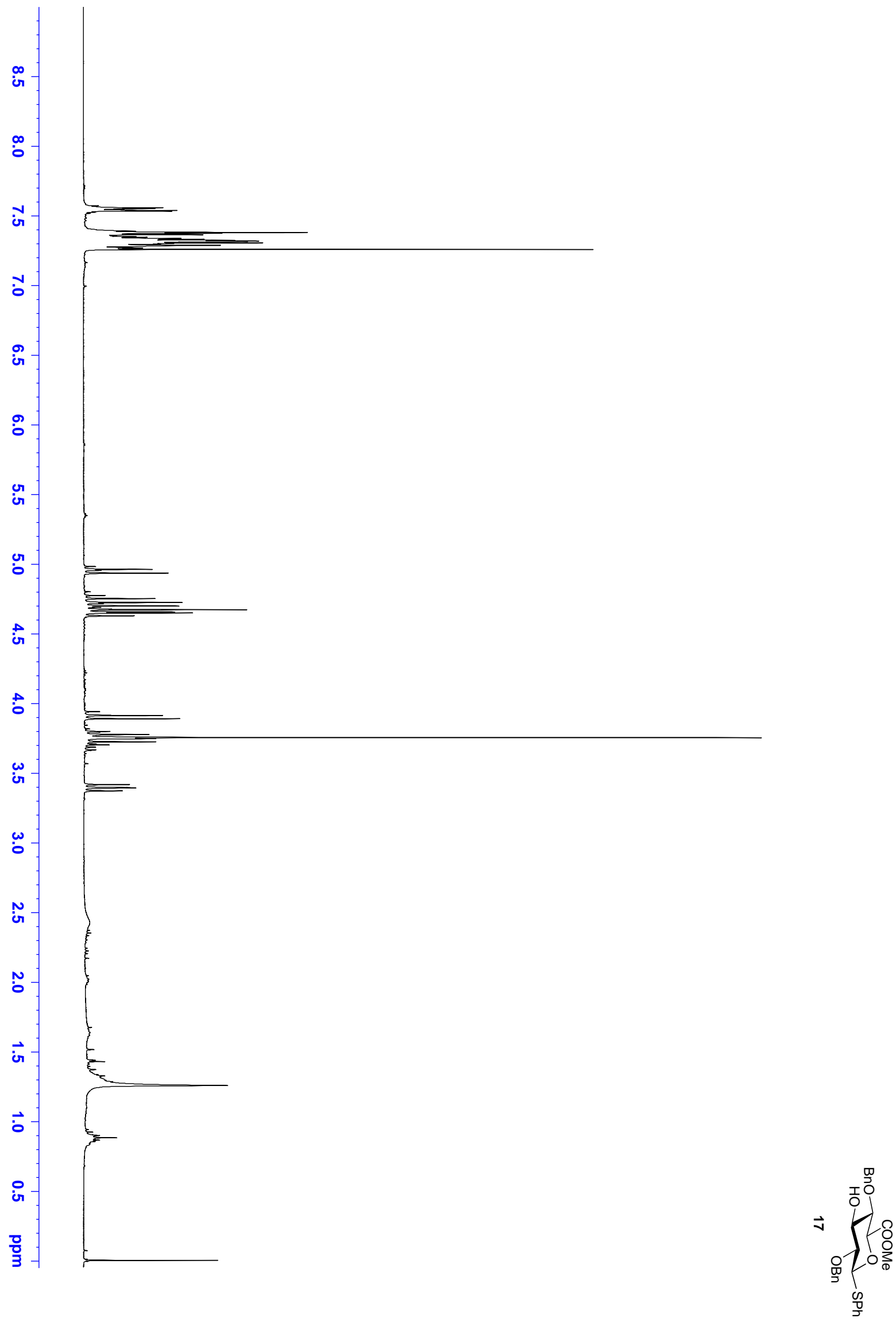

26 of 43 


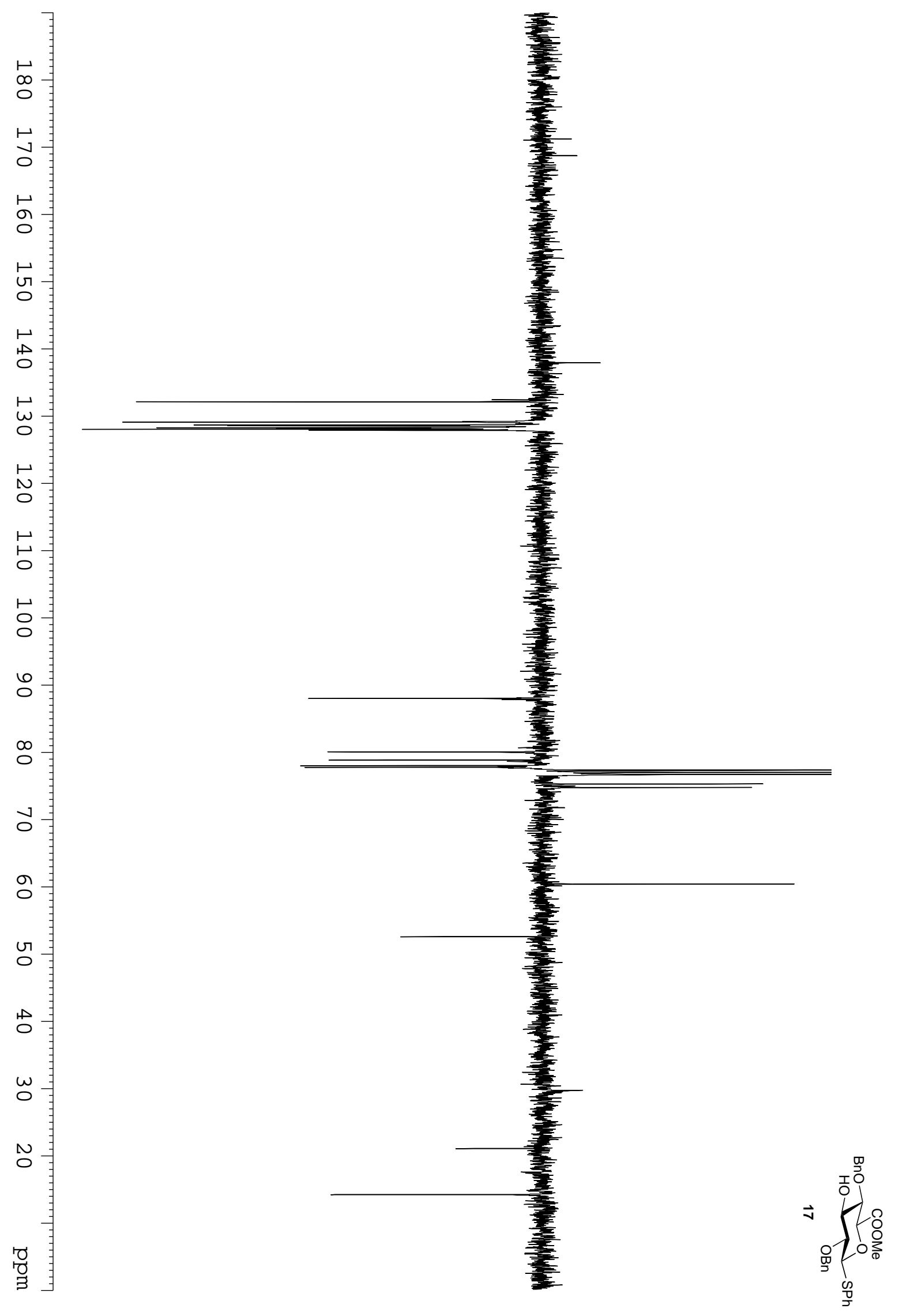

27 of 43 


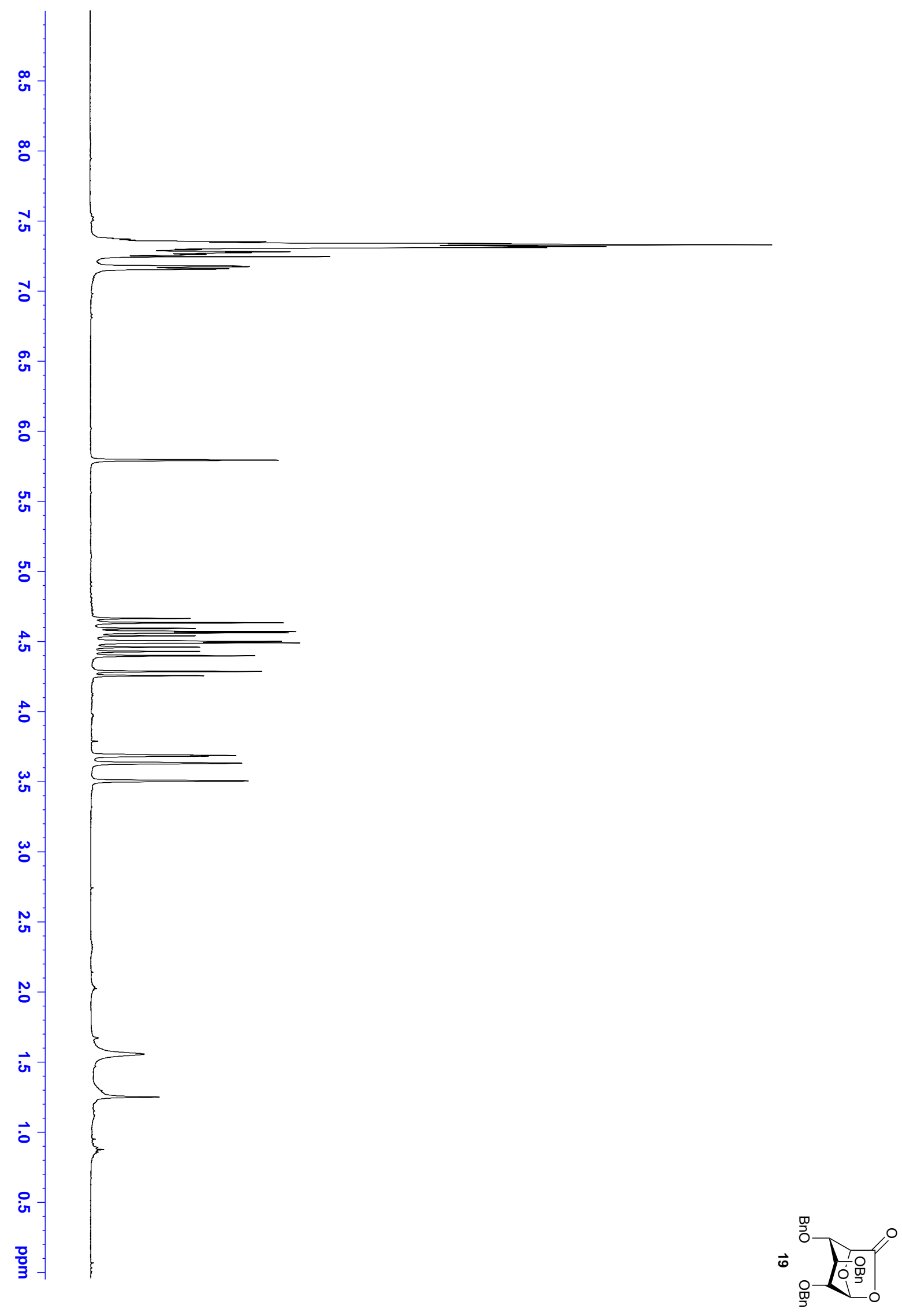




$$
\text { H. }
$$



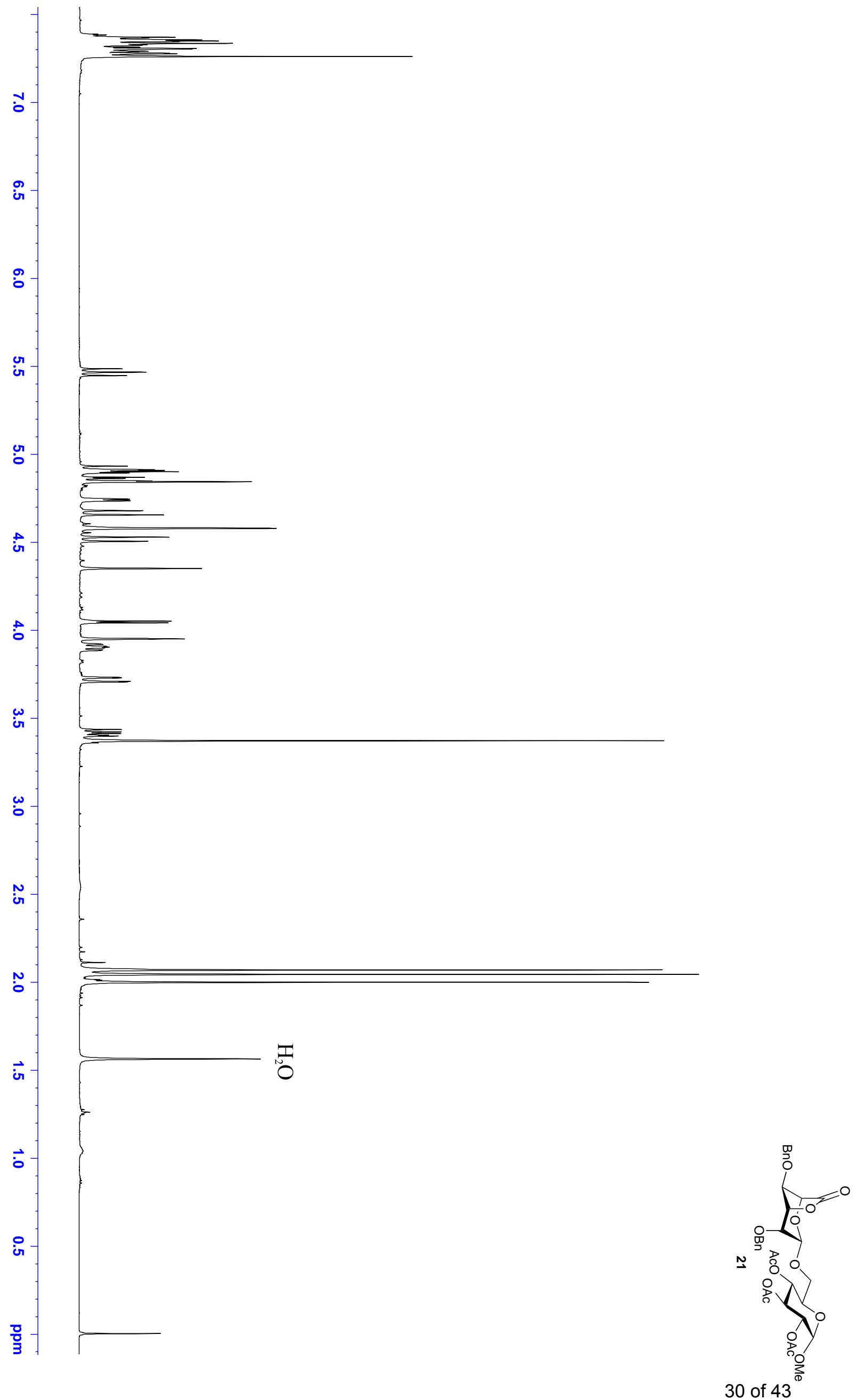


$$
\text { f }
$$



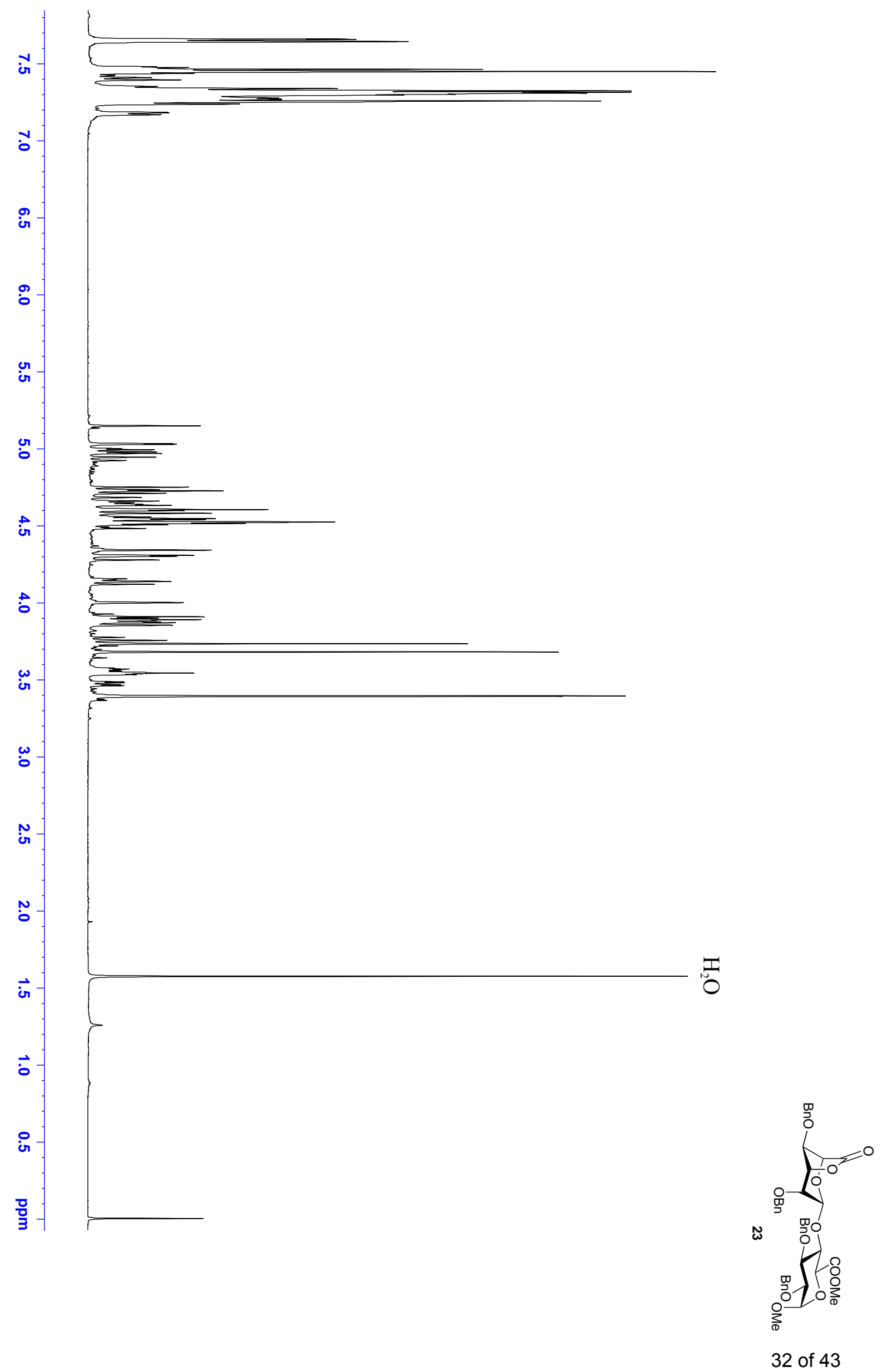


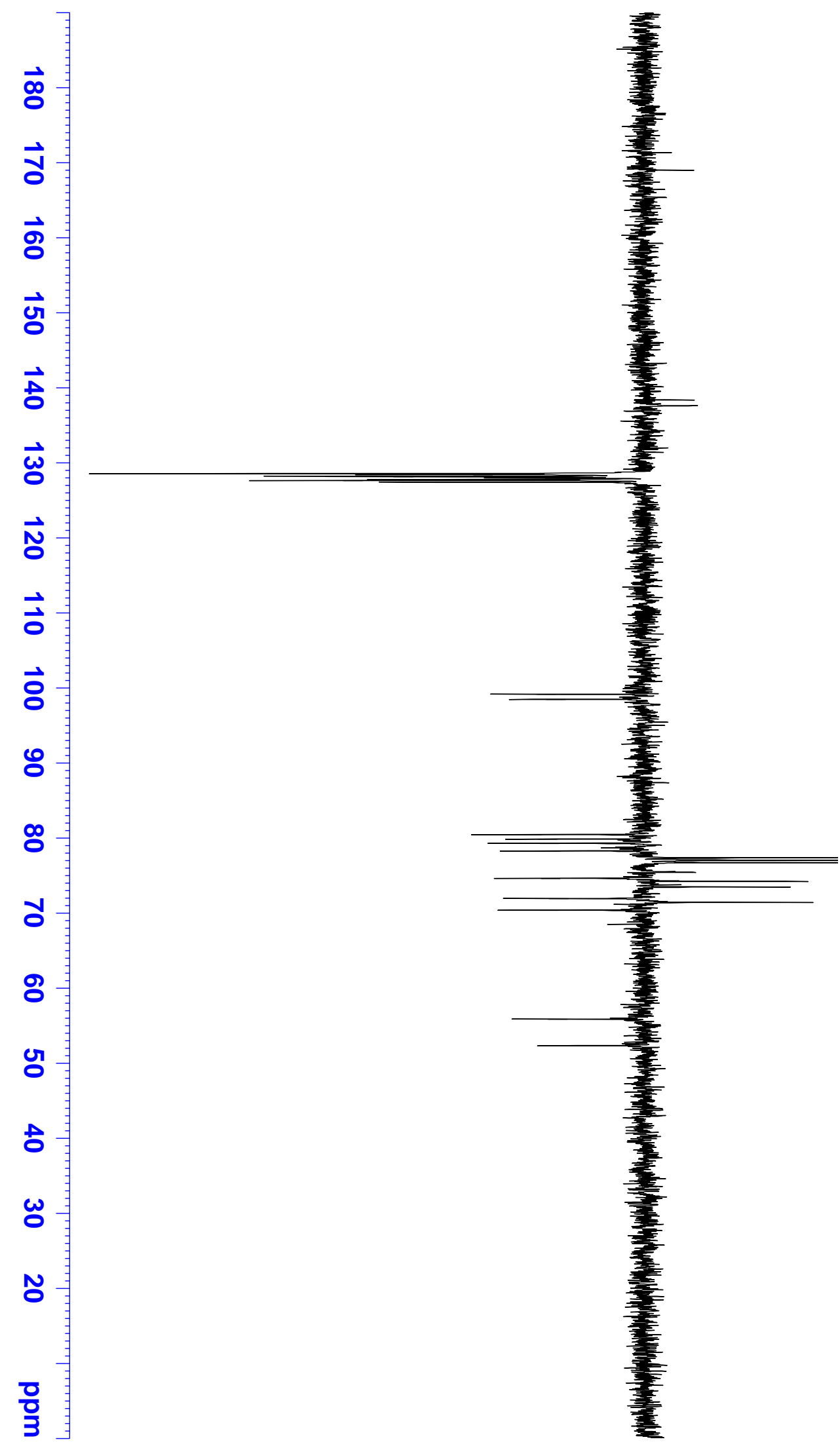

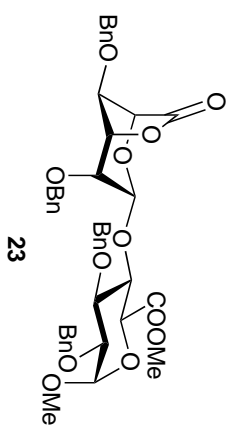

33 of 43 

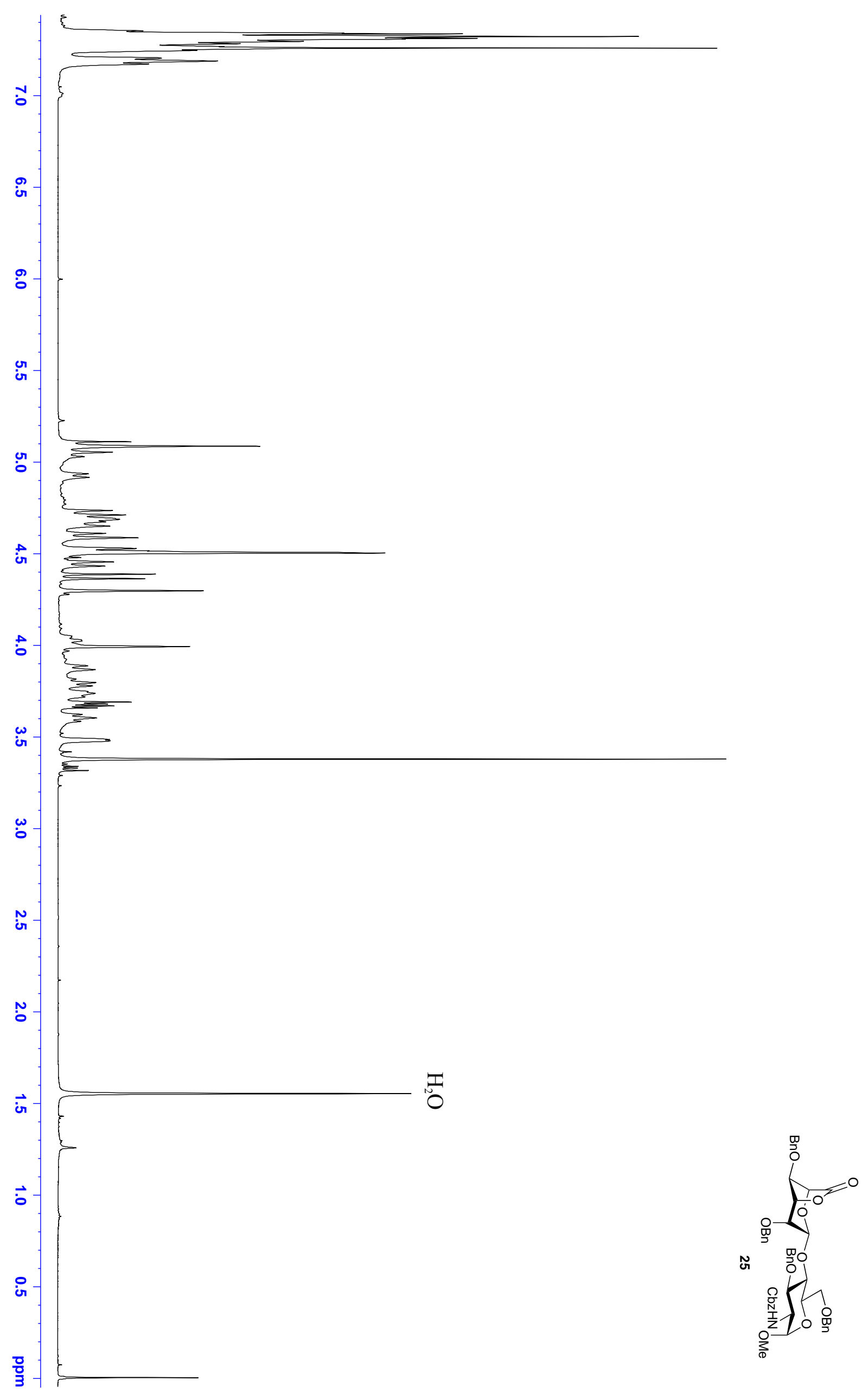

34 of 43 


$$
7 \text {. }
$$




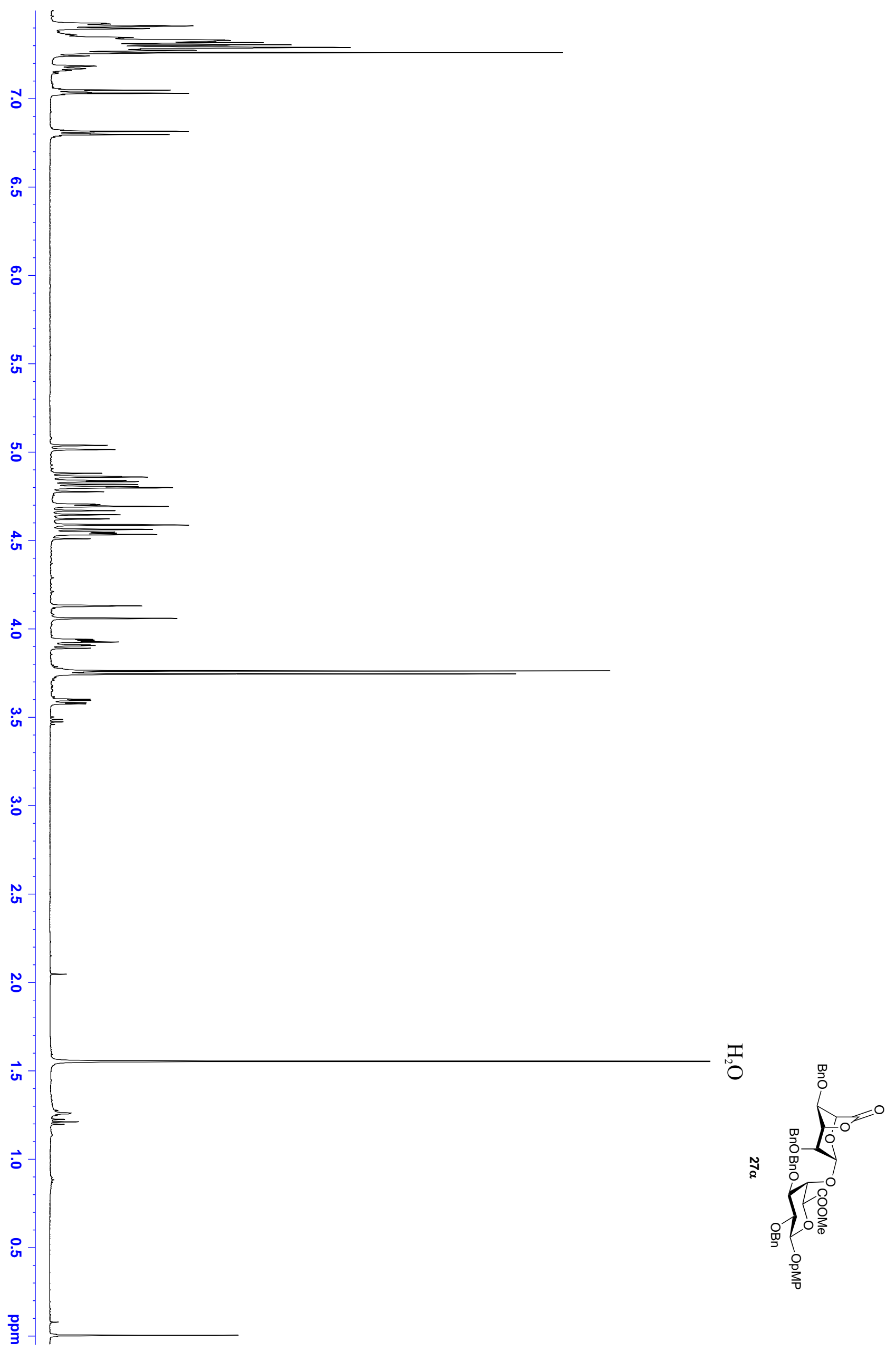




$$
1
$$




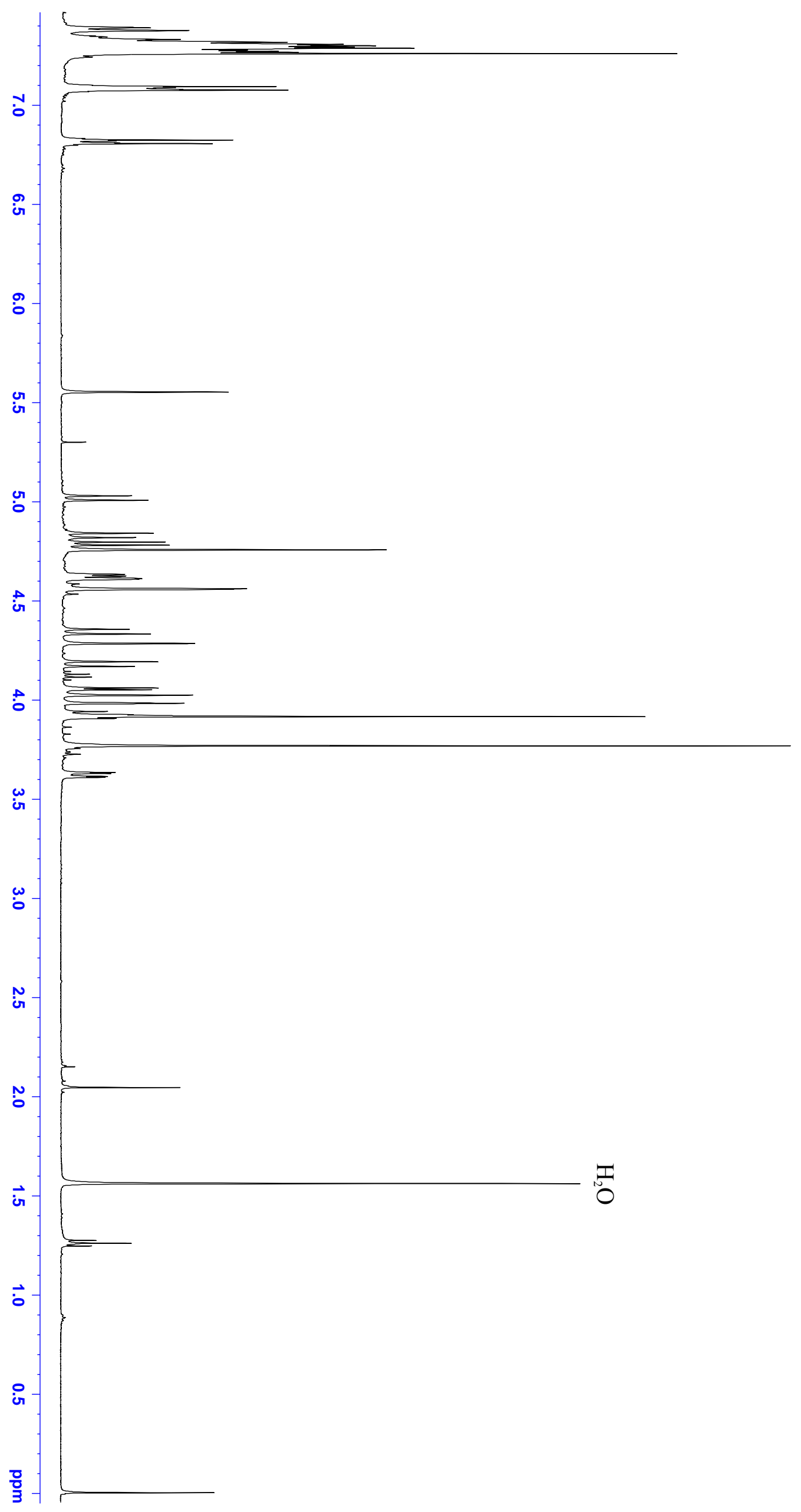

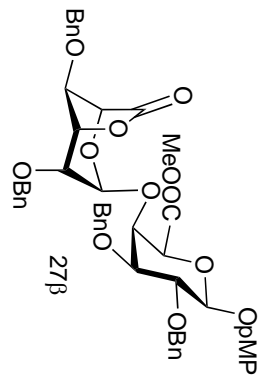

38 of 43 


$$
=
$$




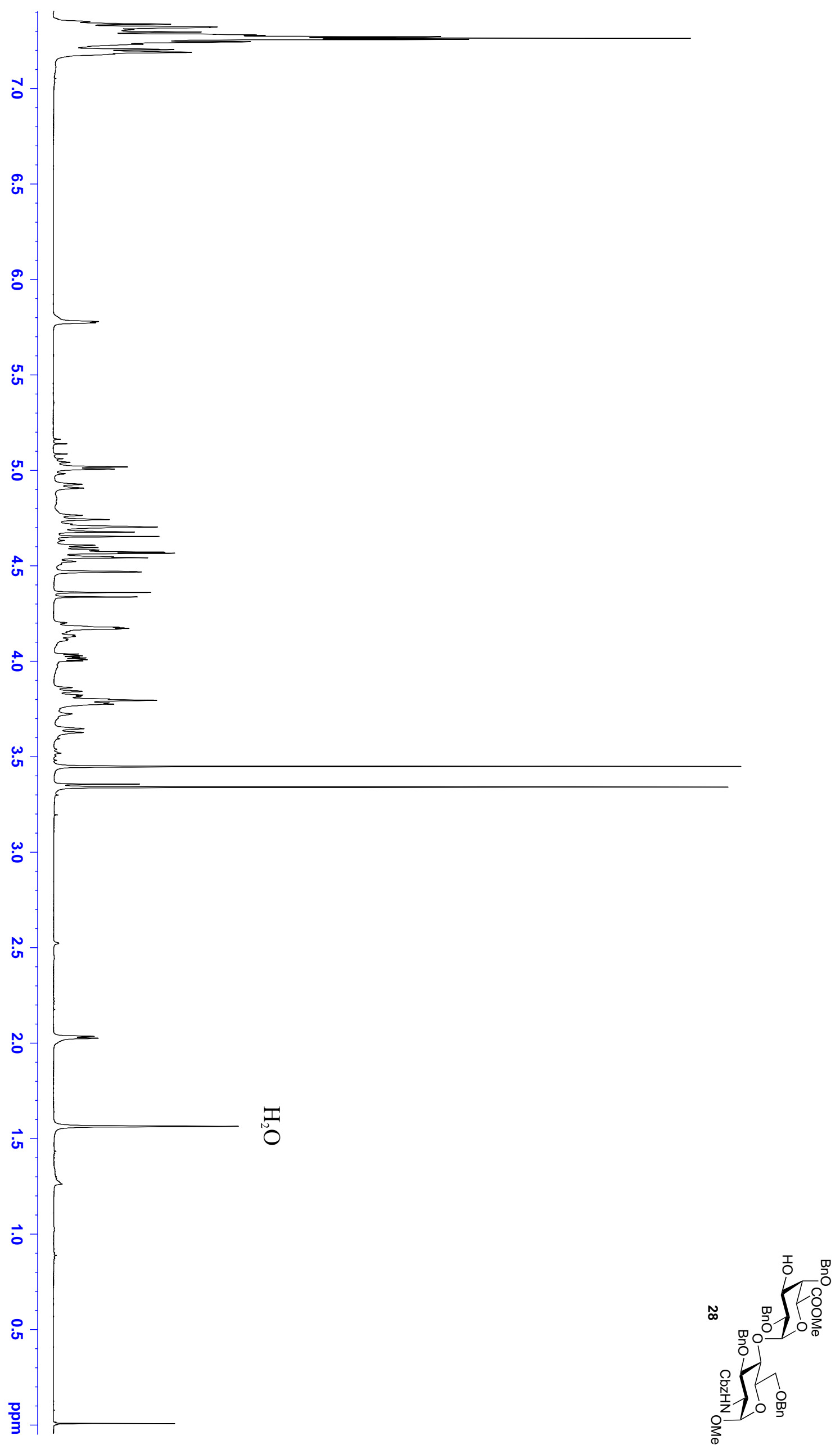




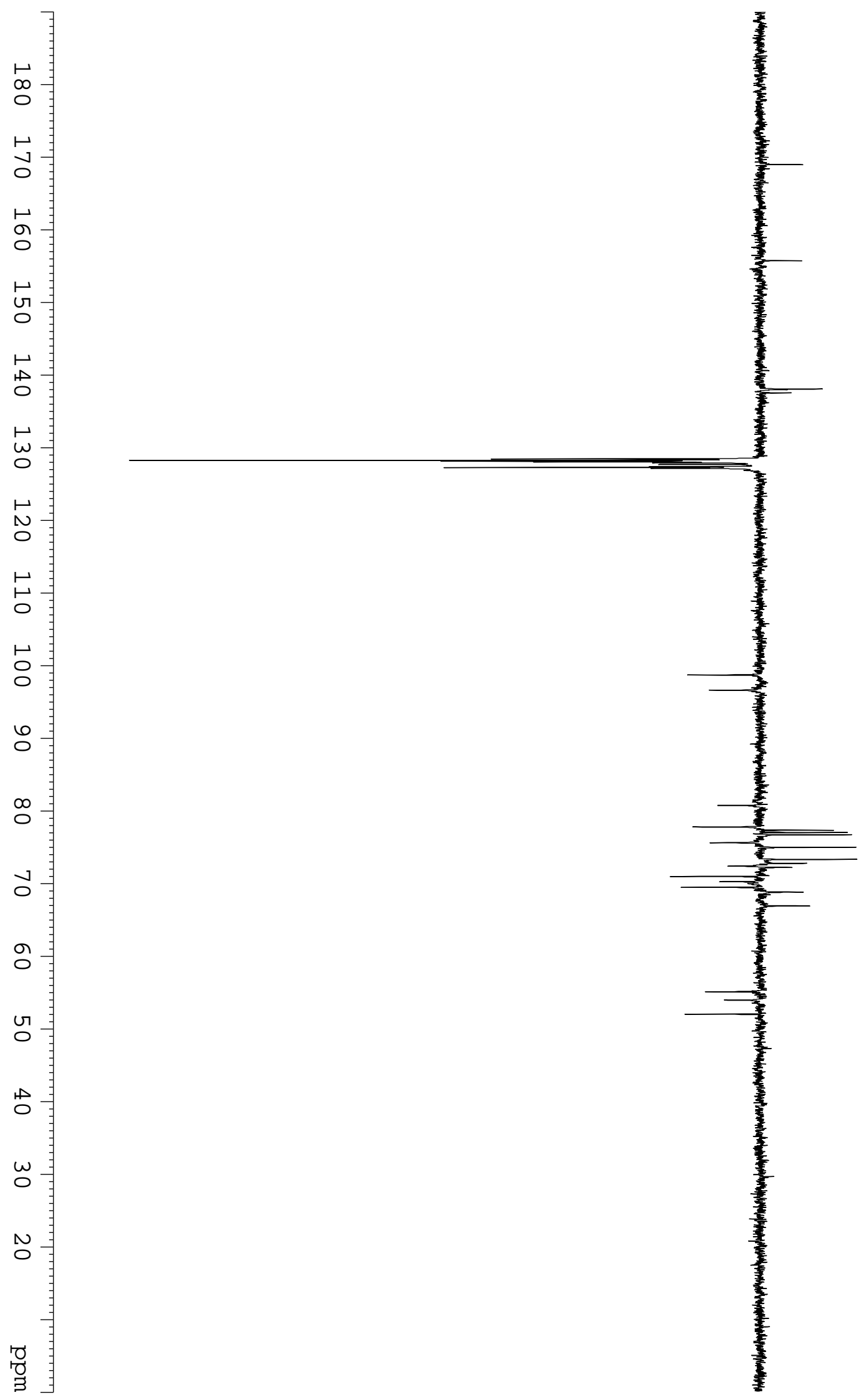

(1) 

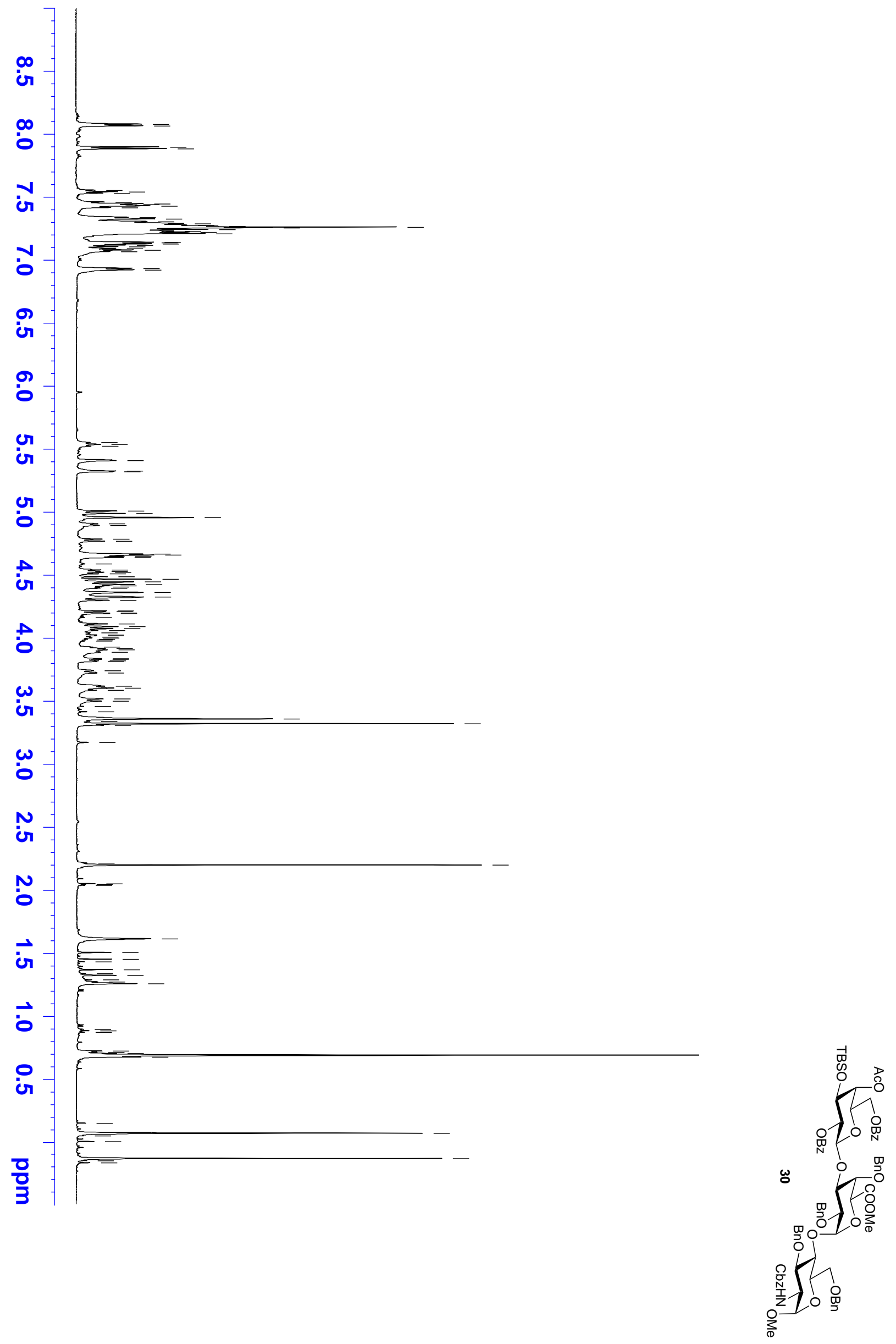

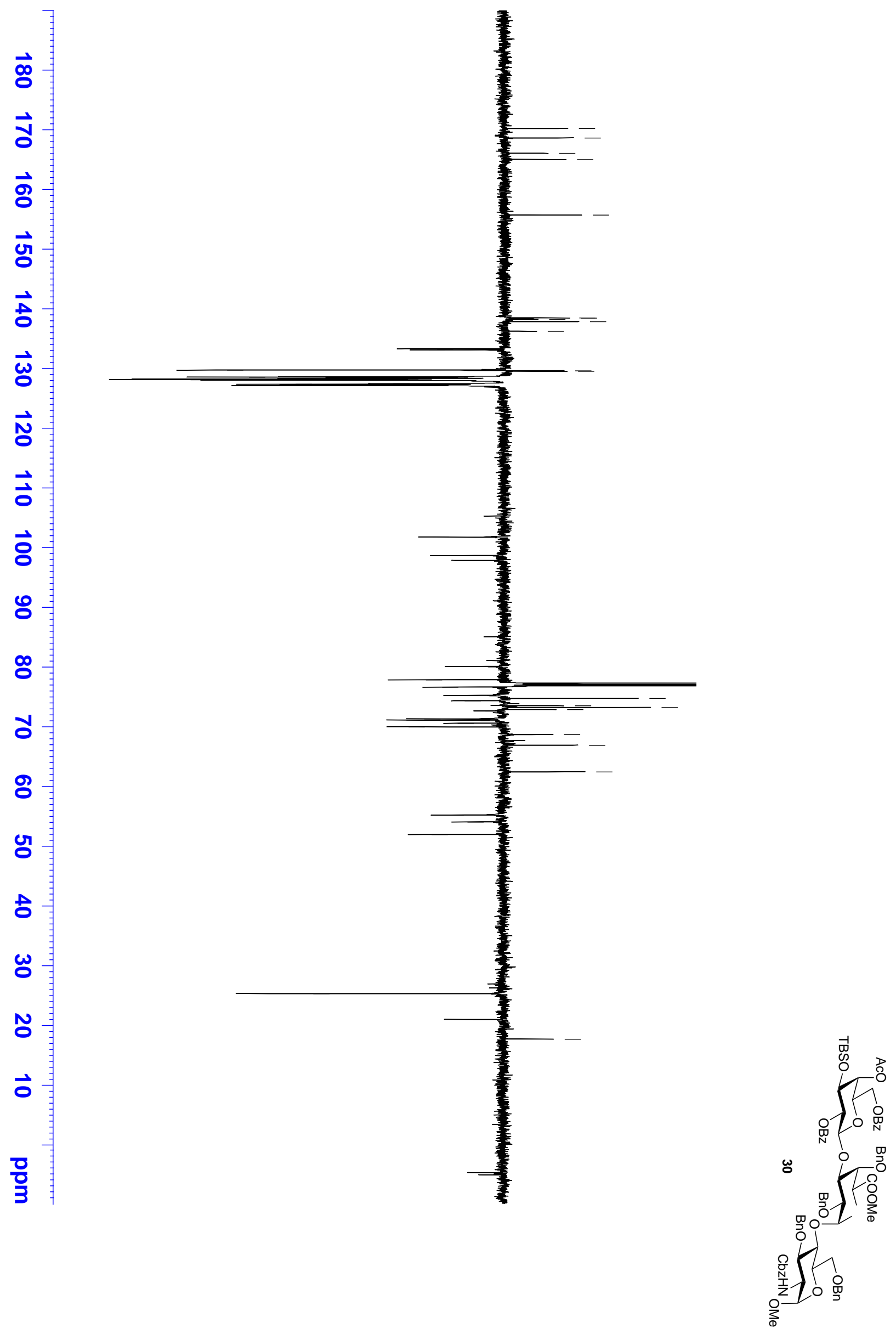

43 of 43 